\title{
Old age causes de novo intracortical bone remodeling and porosity in mice
}

\author{
Marilina Piemontese, ${ }^{1}$ Maria Almeida, ${ }^{1}$ Alexander G. Robling, ${ }^{2}$ Ha-Neui Kim, ${ }^{1}$ Jinhu Xiong, ${ }^{1}$ \\ Jeff D. Thostenson, ${ }^{3}$ Robert S. Weinstein, ${ }^{1}$ Stavros C. Manolagas, ${ }^{1}$ Charles A. O'Brien, ${ }^{1}$ \\ and Robert L. Jilka1 \\ ${ }^{1}$ Center for Osteoporosis and Metabolic Bone Diseases, University of Arkansas for Medical Sciences, and the Central \\ Arkansas Veterans Healthcare System, Little Rock, Arkansas, USA. ²Department of Anatomy and Cell Biology, Indiana \\ University School of Medicine, Indianapolis, Indiana, USA. ${ }^{3}$ Department of Biostatistics, University of Arkansas for Medical \\ Sciences, Little Rock, Arkansas, USA.
}

\begin{abstract}
Decreased cortical thickness and increased cortical porosity are the key anatomic changes responsible for osteoporotic fractures in elderly women and men. The cellular basis of these changes is unbalanced endosteal and intracortical osteonal remodeling by the osteoclasts and osteoblasts that comprise the basic multicellular units (BMUs). Like humans, mice lose cortical bone with age, but unlike humans, this loss occurs in the face of sex steroid sufficiency. Mice are therefore an ideal model to dissect age-specific osteoporotic mechanisms. Nevertheless, lack of evidence for endosteal or intracortical remodeling in mice has raised questions about their translational relevance. We show herein that administration of the antiosteoclastogenic cytokine osteoprotegerin to Swiss Webster mice ablated not only osteoclasts, but also endosteal bone formation, demonstrating the occurrence of BMU-based endosteal remodeling. Femoral cortical thickness decreased in aged male and female C57BL/6) mice, as well as $\mathrm{F} 1$ hybrids of $\mathrm{C57BL} / 6$ ] and $\mathrm{BALB} / \mathrm{CBy}$ mice. This decrease was greater in $\mathrm{C57BL} / 6 \mathrm{~J}$ mice, indicating a genetic influence. Moreover, endosteal remodeling became unbalanced because of increased osteoclast and decreased osteoblast numbers. The porosity of the femoral cortex increased with age but was much higher in females of both strains. Notably, the increased cortical porosity resulted from de novo intracortical remodeling by osteon-like structures. Age-dependent cortical bone loss was associated with increased osteocyte DNA damage, cellular senescence, the senescence-associated secretory phenotype, and increased levels of RANKL. The demonstration of unbalanced endosteal and intracortical remodeling in old mice validates the relevance of this animal model to involutional osteoporosis in humans.
\end{abstract}

Conflict of interest: SCM is a cofounder and owns equity of Radius Health Inc.

Submitted: March 6, 2017

Accepted: August 3, 2017

Published: September 7, 2017

\section{Reference information:}

JCI Insight. 2017;2(17):e93771. https:// doi.org/10.1172/jii.insight.93771.

\section{Introduction}

The increased incidence of osteoporotic fractures in elderly women and men is due to a large extent to structural deterioration of cortical bone, which represents about $80 \%$ of the skeleton $(1,2)$. Specifically, cortical thickness decreases and porosity increases, as determined by analysis of postmortem specimens and high-resolution peripheral quantitative CT (HRpQCT) imaging of the distal radius (2-8). These changes occur in both sexes but are greater in women $(5,6)$.

In humans and other large mammals, cortical bone is composed of circumferential lamellae interrupted by numerous osteons - cylindrical concentric layers of bone surrounding a vascular channel called the Haversian canal (9). The integrity of cortical bone is maintained by teams of osteoclasts and osteoblasts supplied via the vascular channel and collectively known as the basic multicellular unit (BMU). The BMU tunnels through the cortex and replaces old bone with new, thus generating a new osteon with embedded osteocytes connected to one another and to the central capillary via a lacuno-canalicular system. The size of the canals, and therefore porosity, increases with age because the bone removed by resorption is not fully replaced (10). Repeated episodes of unbalanced remodeling cause the enlargement and merging of the canals. The interconnectivity of the vascular channels also increases with age (11), and merged osteons are evident at sites where closely spaced clusters of osteons develop from capillary branches of the vascular channel (12-14). Together with unbalanced remodeling of the endosteal surface $(15,16)$, these events transform the cortex 
A

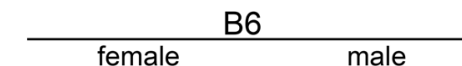

femur

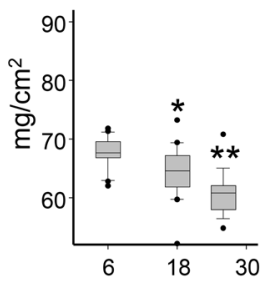

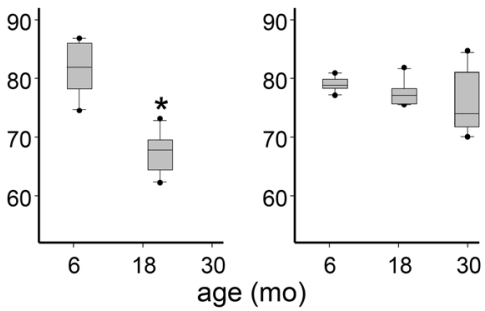

$\mathrm{CB} 6 \mathrm{~F} 1$

female

male

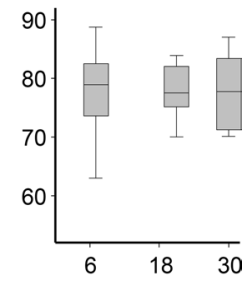

Figure 1. Age-related bone loss is greater in B6 than in CB6F1 mice. Bone mineral density was determined in the (A) femur, (B) lumbar vertebrae, and (C) total body excluding the head. Number of mice analyzed, ordered by increasing age: female $\mathrm{B} 6(20,20,19)$, male $\mathrm{B} 6(10,10)$, female CB6F1 $(10,10,10)$, male CB6F1 $(9,9,8) .{ }^{*} P<0.05$ vs. 6 - to 7-month-old sex-matched animals of the same strain, ${ }^{* *} P$ $<0.05$ vs. 6 - to 7 -month and 18 - to 19 -month sex-matched animals of the same strain, as determined by two-tailed $t$ test (B6 males) or one-way ANOVA (B6 females and CB6F1 females and males).

B lumbar vertebrae
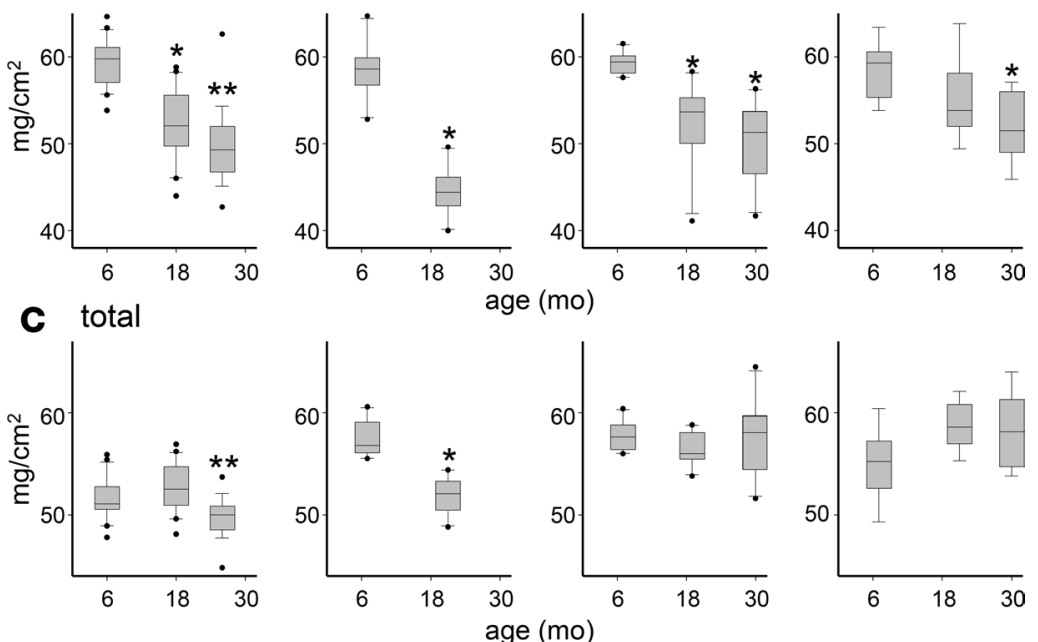

adjacent to the BM into a structure resembling trabecular bone. Heretofore, the underlying mechanisms responsible for the loss of cortical bone with age remain unknown.

Observations made in rodents have provided invaluable insights into bone physiology and pathophysiology, and discoveries from rodents, complemented with human studies, have led to the development of both antiresorptive and anabolic therapies for osteoporosis (17). Like humans, mice lose cortical bone with age. Femoral growth ceases at $\sim 6-7$ months in C57BL/6J (B6) mice. At $\sim 12$ months (equivalent to 40 years in humans; ref. 18), the marrow begins to expand, and additional bone is slowly added to the periosteum; however, the former exceeds the latter, leading to a thinner and more fragile cortex (19-22). Aging female mice do not experience menopause but become acyclic while retaining functional levels of estrogens $(17,23)$, and androgen levels in aged male mice are maintained at a 20 -fold higher level than in females (24). However, both female and male mice exhibit all of the major features of skeletal aging, including the decline of cortical bone mass and the development of cortical porosity by 18 months of age, independently of sex steroid deficiency and by mechanisms that are distinct from those of sex steroid deficiency (23). Thus, mice are an invaluable model for dissecting the mechanisms of skeletal aging per se from the effects of sex steroid deficiency. However, it remains unclear whether age-dependent marrow expansion and cortical thinning with age in mice results from unbalanced remodeling of the endosteal surface or from osteoclastic modeling that is not linked to osteoblastic bone formation. Similar to humans, increased femoral cortical porosity has been reported in $\sim 2$-year-old male B6 mice and in female mice with a mixed genetic background $(21,25)$; however current dogma holds that osteonal remodeling does not occur in rodents (26). This situation has raised concerns about the relevance of the mouse model to our understanding of mechanisms responsible for the loss of cortical bone with age in humans.

To gain a better appreciation of age-dependent loss of cortical bone in mice, we quantified the architectural changes that occur in female and male $\mathrm{B} 6$ mice, as well as $\mathrm{CB} 6 \mathrm{~F} 1$ mice that are first-generation progeny of BALB/cBY females and B6 males. Notably, BALB/cBy mice maintain cortical bone mass until 20 months of age (27). CB6F1 mice are genetically identical to one another but have alternative alleles for genes that differ in the parental strains, which are distantly related (28). We also investigated the cellular 
A

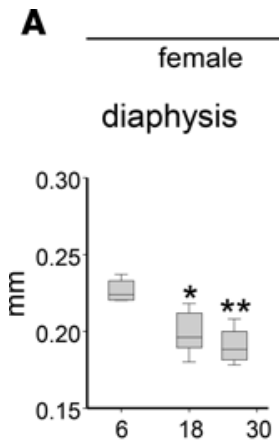

B6

male

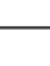
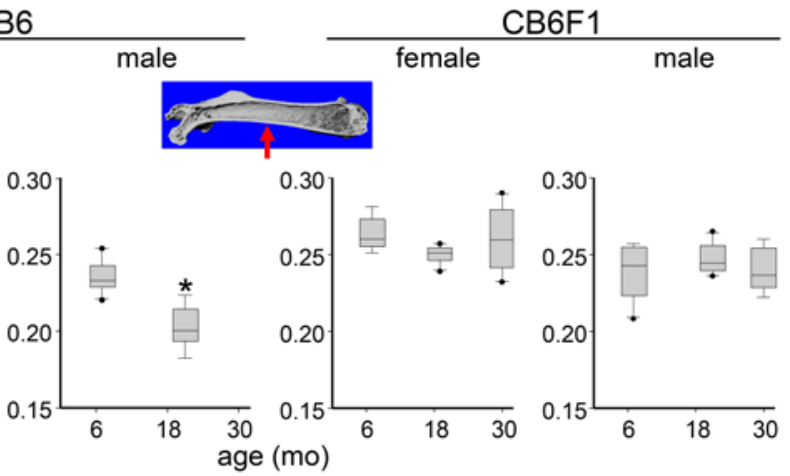

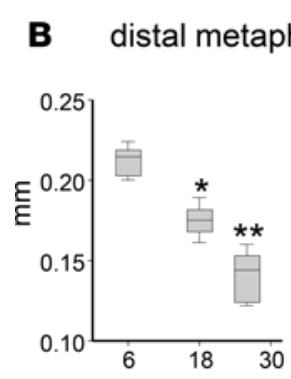

\section{ysis}
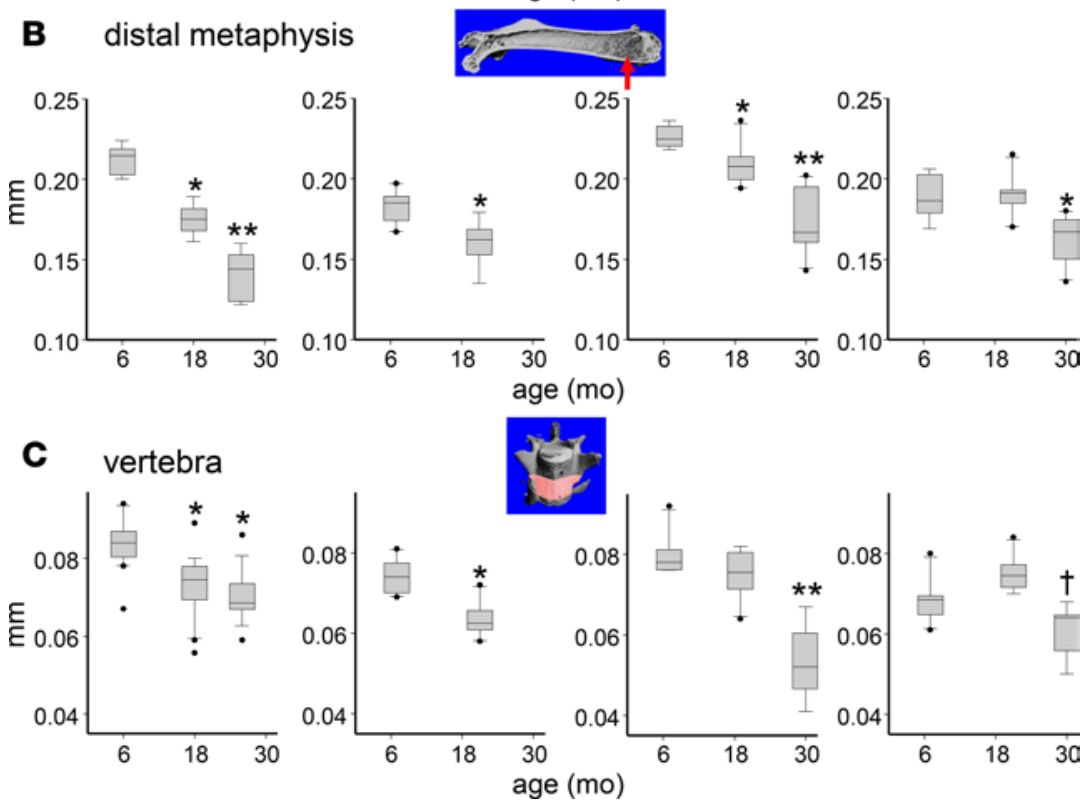

age (mo)

Figure 2. Age-related decrease in cortical thickness is greater in B6 than in CB6F1 mice. Cortical thickness was determined at the (A) mid-diaphysis and (B) distal metaphysis of the femur, as well as at the (C) anterior vertebra (L4), as indicated by the red arrows and pink fill in the microCT images. Number of mice analyzed, ordered by increasing age: female $\mathrm{B} 6(8,8,7)$, male $\mathrm{B} 6$ $(10,10)$, female $\operatorname{CB} 6 F 1(8,10,10)$, male CB6F1 $(9,9,8)$; $n=5$ for metaphyseal cortical thickness of 26 -month female B6 mice because of trabecularization of the endosteal boundary. ${ }^{*} P<0.05$ vs. 6 - to 7 -month-old sex-matched animals of the same strain, ${ }^{* *} P<0.05$ vs. 6- to 7-month and 18- to 19-month sex-matched animals of the same strain, $\dagger P<0.05$ vs. 19 -month sex-matched animals of the same strain, as determined by two-tailed $t$ test (B6 males)or one-way ANOVA (B6 females and CB6F1 females and males).

basis of cortical porosity in B6 mice and the impact of aging on cortical osteocytes because of emerging evidence for a role of these cells in normal and pathologic bone remodeling $(29,30)$.

\section{Results}

Age-dependent bone loss differs in B6 and CB6F1 mice. We compared the bone mass of skeletally mature 6-7 month-old mice with the bone mass of 18-30 month-old mice that are in the latter half of their life span (31-33). The bone mineral density (BMD) of the femur of B6 mice, determined by dual-energy x-ray absorptiometry, declined with age in both sexes (Figure 1A). In contrast, femoral BMD of CB6F1 mice did not. Vertebral BMD declined with age in both males and females of both strains (Figure 1B), whereas $\mathrm{BMD}$ averaged over the entire appendicular and axial skeleton fell only in B6 mice (Figure 1C). Body weight increased with age in males and females of both strains by $18 \%-25 \%$ (Supplemental Tables 1 and 2; supplemental material available online with this article; https://doi.org/10.1172/jci.insight.93771DS1). Serum PTH did not change with age in female B6 mice (6 month, $94 \pm 70 \mathrm{pg} / \mathrm{ml}, n=19$ vs. 26 month, $120 \pm 96 \mathrm{pg} / \mathrm{ml}, n=16$ ), indicating that the age-dependent skeletal changes are not caused by primary or secondary hyperparathyroidism.

The age-dependent decline in cortical thickness is greater in B6 than in CB6F1 mice. Microcomputed tomography (microCT) showed that the thickness of the femoral diaphysis of B6 mice was lower in 19- and 30-month-old mice, as compared with 6- to 7-month-old mice in both sexes (Figure 2A). In contrast, aging had no detectable impact on the cortical thickness of the diaphysis in female or male CB6F1 mice. However, at the distal metaphysis, cortical thickness was lower in 19- to 30-month females and males of both strains, as compared with young adults (Figure 2B). This decline was evident at 21 months in male B6 mice but was not observed until 30 months of age in male CB6F1 mice. Both strains exhibited marrow expansion with age, as determined by increased medullary area measured at the diaphysis and, except for B6 males, at the metaphysis (Supplemental 
A

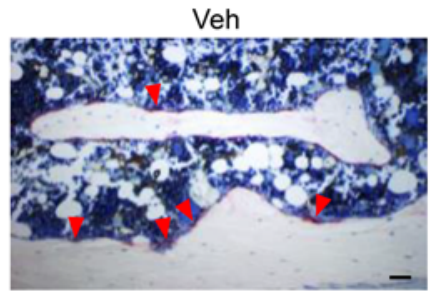

B
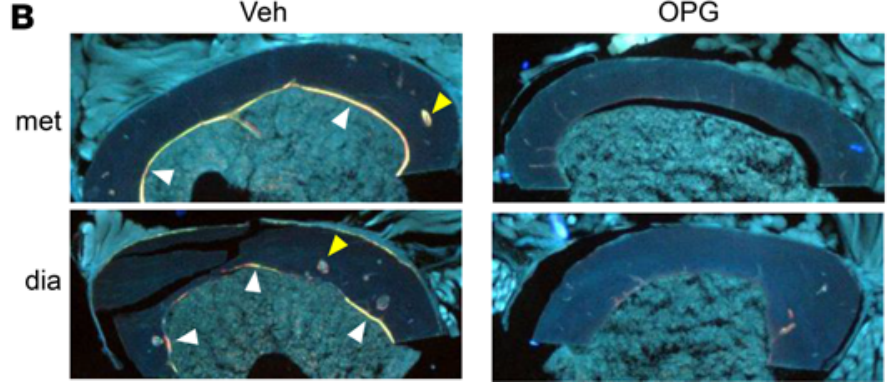

Figure 3. Osteoprotogerin eliminates endosteal bone formation in adult mice. Female 7-month-old Swiss-Webster mice were injected with vehicle (Veh) or OPG. (A) Longitudinal sections of the femur stained for TRAP to visualize osteoclasts (red arrowheads). Scale bar: $20 \mu \mathrm{m}$. (B) Unstained cross-sections made at the distal metaphysis (met) and middiaphysis (dia) to visualize alizarin (red) and calcein (yellow) fluorochromes. White arrowheads, sites of endosteal bone formation; yellow arrowhead, site of bone formation within a cortical pore. Original magnification: $40 x$.
Tables 3 and 4). Periosteal expansion, as measured by total area at the diaphysis, occurred with age in both strains but was less evident at the metaphysis. Since the femur of adult mice mainly comprises cortical bone, the smaller change in cortical thickness in aging CB6F1 probably explains the lack of change in femoral BMD with age in this strain (Figure 1A). The thickness of the cortical bone of lumbar vertebra L4 was also lower in 16- to 26-month female and 21-month male B6 mice as compared with the 6- to 7-month mice of the same sex; however, this decrease could not be detected in female or male CB6F1 mice until 30 months of age (Figure 2C).

Both male and female B6 and CB6F1 mice lose trabecular bone with age. Consistent with earlier studies showing rapid loss of trabecular bone of the distal femur between 2 and 6 months in female and male $\mathrm{B} 6$ mice (34), trabecular bone volume (BV/TV) was very low $(\sim 6 \%)$ at this site in females of both strains at 6-7 months of age. At 6-7 months of age, BV/TV was 2- to 3-fold higher in males than in females in both strains, but it decreased with age in both sexes of both strains due to a decrease in trabecular number and an increase in trabecular separation (Supplemental Tables 5 and 6).

Vertebral BV/TV progressively declined with age in males and females of both strains, due mainly to reduced trabecular number (Supplemental Tables 5 and 6). A decrease in trabecular thickness was also detected in 26-month-old B6 and 30-monthold CB6F1 females, as compared with 6- to 7-month-old young adults. These findings are consistent with the age-dependent fall in vertebral bone mass noted in Figure 1B.

Cortical thinning with age is due to unbalanced endosteal remodeling. Age-dependent medullary expansion, and therefore cortical thinning, in adult mice is due to either osteoclastic modeling or unbalanced BMUbased remodeling of the endocortical surface (35). The latter possibility is supported by previous studies in adult rats showing that sites of endosteal bone formation are juxtaposed to scalloped cement lines that mark sites of previous episodes of bone resorption (36). To firmly establish the coupling of endosteal bone formation to bone resorption in adult mice, we determined the effect of osteoprotogerin (OPG) - an antagonist of the RANKL required for the formation and survival of osteoclasts - on endosteal bone formation. As expected, administration of OPG to 7-month-old female Swiss-Webster mice for 19 days eliminated osteoclasts from the endosteal surface of the diaphyseal and metaphyseal cortexes (Figure 3A). More importantly, OPG also eliminated endosteal fluorochrome labeling that marks sites of osteoblastic bone formation (Figure 3B). This result demonstrates that the endosteal surface of adult mice indeed undergoes BMU-based remodeling.

We next investigated the distribution of BMUs along the endosteal surface of adult mice. To do this, we used female B6 mice that had been given fluorochromes during growth $(0.6,1.4$, and 2.6 months of age) and at 9.5 and 10.7 months of age, as depicted in the left panel of Figure 4A, and then euthanized at 11 months of age. The latter two labels will specifically identify endosteal sites of recent BMU activity. Discrete endosteal fluorochrome labeling was observed in cross-sections made at the proximal, diaphyseal, and distal metaphyseal femur (Figure 4, B-D, respectively). Besides isolated labels, adjacent and overlapping labels were observed (Figure 4E), suggesting repeated remodeling of the same surface during the 6 weeks prior to euthanasia. Endosteal labels were present in medial, proximal, lateral, and anterior sites of each section. Comparison of the location of endosteal labeling in 2 mice suggests that BMUs are randomly located. In contrast, the location of fluorescent labels administered during growth exhibited a predictable pattern. Thus, smooth labels in the interior of the cortex marked sites of periosteal apposition (green-red-orange proceeding from the endosteum), which are particularly evident in the proximal femur. At the diaphysis, cortical drift (37) accounts for lack of significant red labeling and the reversal of labeling order to orange-red in the anterior portion. The filigreed pattern of green labels within the medial portion of diaphyseal cortex, and similarly shaped orange and red labels in the distal metaphyseal cortex, reflect bone formation in trabeculae that were subsequently incorporated into cortical bone during growth (37-39). 
A

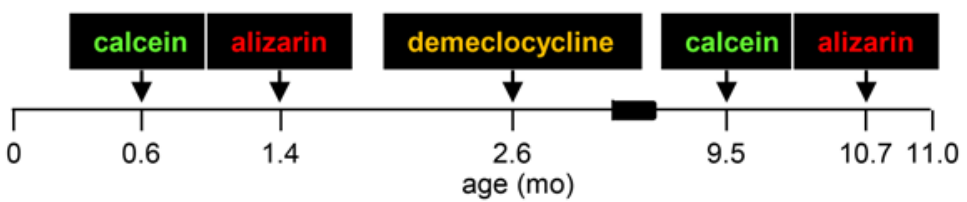

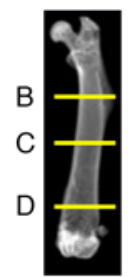
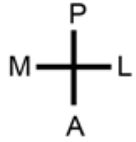

\section{B}
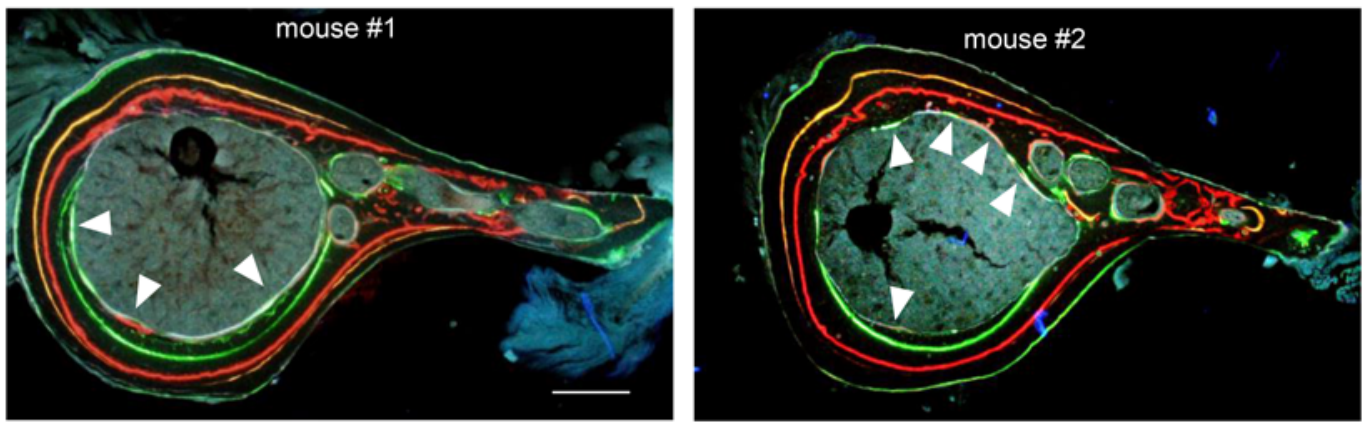

C
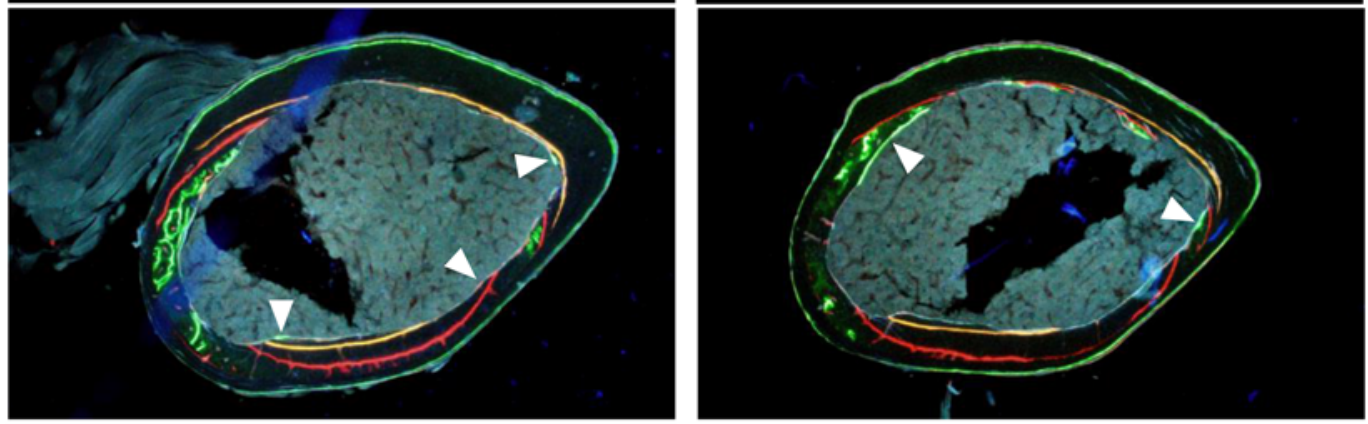

D
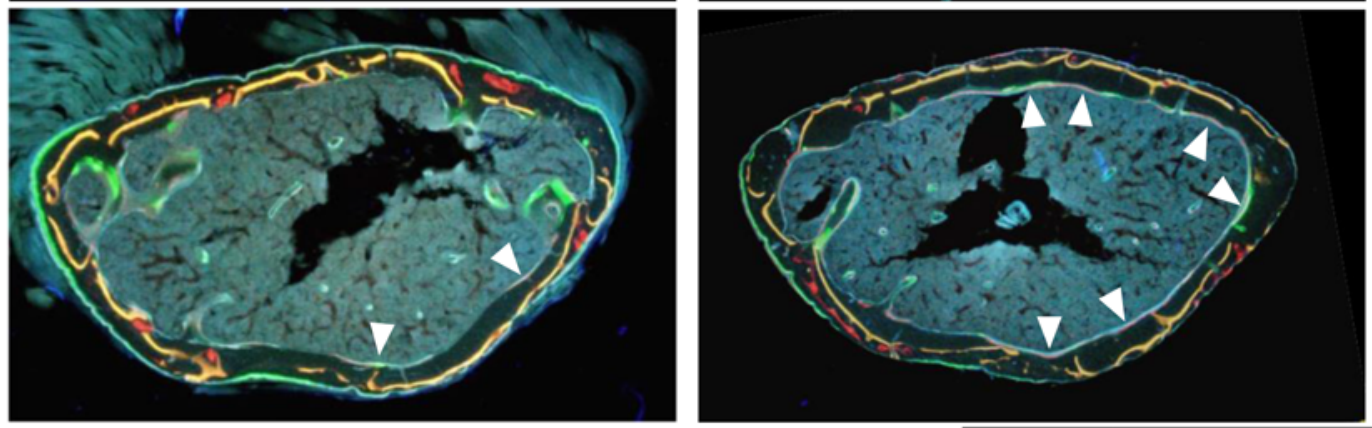

$\mathbf{E}$
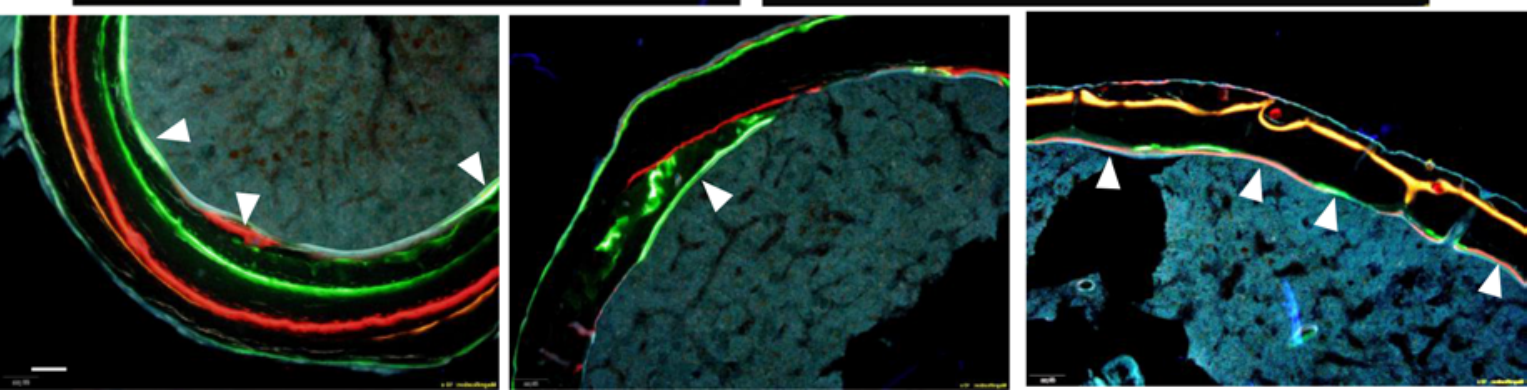

Figure 4. Multiple fluorochrome labeling of femoral bone during growth and adulthood. Female B6 mice were given calcein, alizarin, and demeclocycline at the indicated ages (A, left hand panel). Nondecalcified cross sections were prepared at 11 months of age from the (B) proximal, (C) diaphyseal, and (D) distal metaphyseal parts of the femur as indicated (A, middle panel). Photomicrographs from two mice, shown side-by-side (B-D), are oriented as illustrated (A, right panel); M, medial; P, posterior; L, lateral; A, anterior. Scale bar: $200 \mu \mathrm{m}$. (E) Representative high-power images from proximal (left), diaphyseal (middle), and metaphyseal (right) cross sections. White arrowheads, sites of endosteal bone formation. Scale bar: $50 \mu m$. 
Table 1. Empty osteocyte lacunae are lower in periosteal than in endosteal portion cortical bone of 21-month-old female B6 mice

\begin{tabular}{|c|c|c|c|c|}
\hline & \multicolumn{2}{|c|}{7 month $(n=9)$} & \multicolumn{2}{|c|}{21 month $(n=10)$} \\
\hline & endosteal & periosteal & endosteal & periosteal \\
\hline B.Ar \% & $97.9 \pm 2.7$ & $2.1 \pm 2.7$ & $78.9 \pm 8.5$ & $21.1 \pm 8.5^{A}$ \\
\hline N.Ot/B.Ar, /mm² & $523 \pm 61$ & n.d. & $529 \pm 55$ & $487 \pm 89$ \\
\hline empty lacunae \% & $3.4 \pm 1.5$ & n.d. & $4.1 \pm 2.5$ & $1.7 \pm 2.2^{B}$ \\
\hline
\end{tabular}

Lamellar periosteal cortex was detectable in 5 of the 7-month-old animals. Empty lacunae in the periosteal cortex were detected in 5 of the 21-month-old animals. ${ }^{A} P<0.05$ vs. 7-month-old sex-matched animals of the same strain by two-tailed $t$ test; ${ }^{B} P<0.05$ vs. 21 -month endosteal cortex by Rank Sum test. B.Ar, bone area, N.Ot, osteocyte number, n.d. not determined.

We further compared endosteal labeling in 6- and 26-month-old female B6 mice. In these mice, tetracycline was administered 7 and 3 days before euthanasia. Discrete labeling was observed in cross sections made at the diaphysis and distal metaphysis at both ages, including a developing packet of endosteal bone containing 2 labels in aged mice (Figure 5A). Packets of endosteal bone of 7- and 21-month-old mice were also delineated (in a separate experiment) by irregular scalloped cement lines that mark the boundary between previous osteoclastic resorption and subsequent bone formation (Figure 5B). Histomorphometric analysis showed that endosteal osteoclast number increased in 21-month-old B6 female mice as compared with 7-month-old B6 female mice, but osteoblast number was unaffected by age (Figure 5, C and D). However, the average width of the packets of endosteal bone, a histologic index of osteoblast number and activity per remodeling episode, was reduced in aged mice (Figure 5E). Age-dependent changes in mineralizing surface, mineral apposition rate, and bone formation rate (Figure $5, \mathrm{~F}-\mathrm{H}$ ) were not detected. Taken together, these findings show that age-dependent cortical thinning in mice is due to unbalanced endocortical remodeling.

Cortical porosity increases with age in females, but not males, in B6 and CB6F1 mice. MicroCT scanning of femora from 6-, 18-, and 26-month-old female B6 mice revealed increased cortical porosity with age (Figure 6A). This phenomenon was most evident in the metaphyseal regions and the third trochanter. Porosity was also detected in the cortex of the tibia and humerus of aged mice (Supplemental Figure 1, A and B). Assembly of sequential microCT images $(6 \mu \mathrm{m} /$ slice $)$ of the distal half of the femur provided a detailed 3 -D view of cortical porosity (Supplemental Videos 1-3). In contrast to 6-month-old mice, pores were evident in bone from 18- and 26-month old mice, becoming less frequent and smaller with increasing distance from the metaphysis. Pores were often connected with each other. In areas with low porosity, the pores were smaller and tended not to be connected to the BM, whereas large pores were usually contiguous with the medullary cavity. In areas of high porosity, the original endosteal boundary was lost, and the cortex was transformed into trabecular bone that sometimes extended into the marrow.

Quantification of porosity of the distal half of the femur, excluding the cortex adjacent to the remnants of the growth plate, demonstrated an increase with advancing age in B6 females (Figure 6B). However, this phenomenon was highly variable, with porosity values ranging from $1 \%-14 \%$ in 26 -month mice. In contrast, porosity in B6 males was not significantly affected by age. An age-dependent increase in porosity in females, but not males, was also observed in CB6F1 mice.

Aging induces de novo osteonal remodeling in the femoral cortex. Cortical bone of 7-month-old B6 females exhibited small pores ranging in size from $0.2 \times 10^{-3}$ to $2.0 \times 10^{-3} \mathrm{~mm}^{2}$ as seen in toluidine blue-stained longitudinal sections (Figure 7, A-C). The small pores represent intracortical capillaries, some of which extend into the marrow. Additionally, other pores were surrounded by small packets of bone that had cement lines around their periphery (Figure 7A). These pores lacked osteoclasts as identified by tartrate-resistant acid phosphatase (TRAP) staining (Figure 7D), and they did not exhibit labeling by tetracycline administered at 7 and 3 days before euthanasia (not shown). In contrast, in the experiment shown in Figure 4 , in which fluorochromes were administered to female B6 mice during growth, small pores frequently exhibited labeling when analyzed at 11 months of age (Figure 7E).

Similar to the situation in B6 mice, only a few pores of the femoral cortex of 7-month-old female Swiss Webster mice had fluorochrome labeling, whether viewed in transverse sections (Figure 3A) or longitudinal sections (Supplemental Figure 2A). OPG eliminated fluorochrome labeling of pores (Supplemental Figure 2B), indicating coupling of bone formation to bone resorption at this site. Taken together, these findings show that the small cortical pores of young-adult mice represent vessels that 

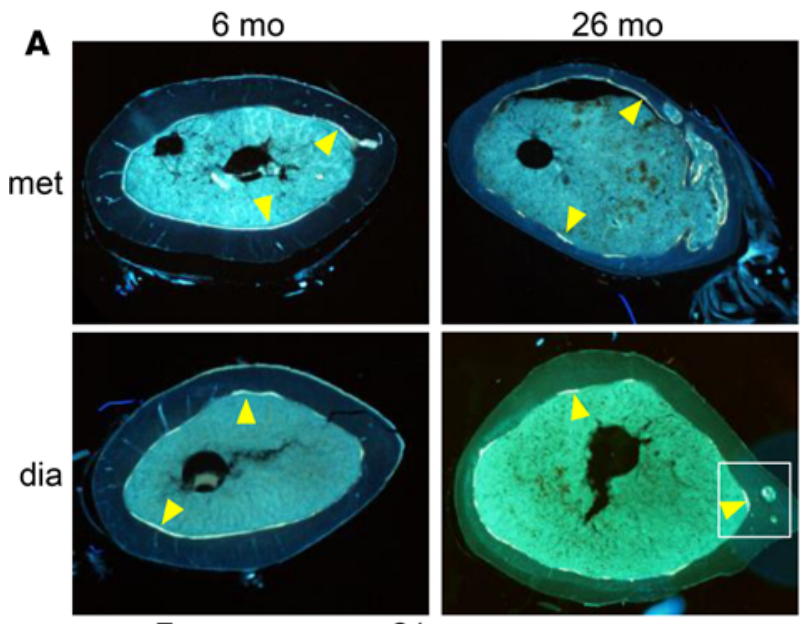

$\mathbf{B}$
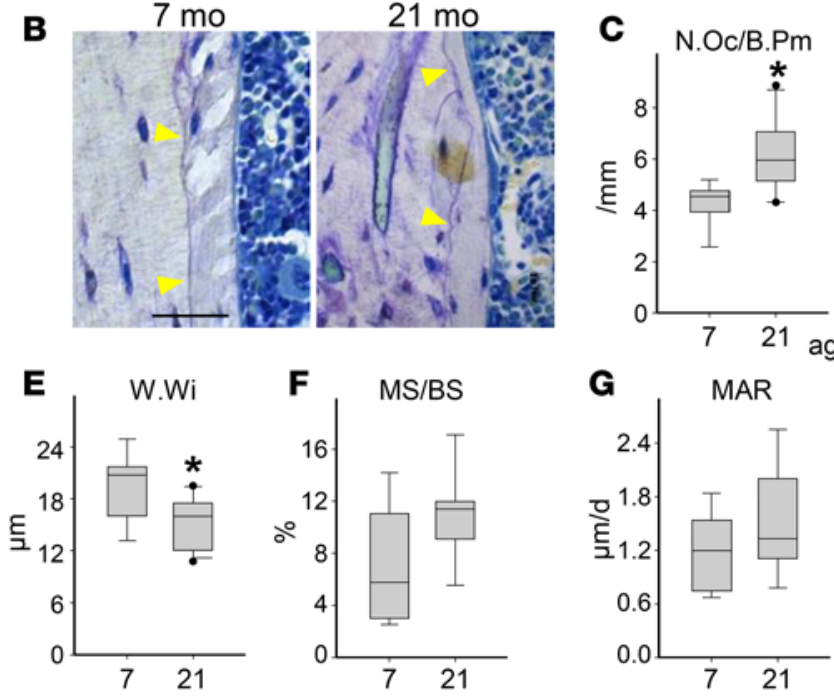

\section{G}

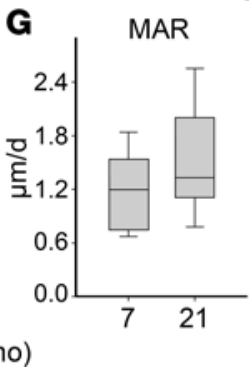

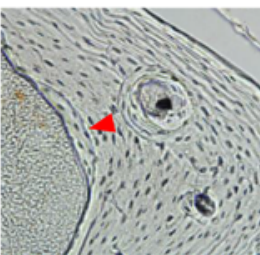
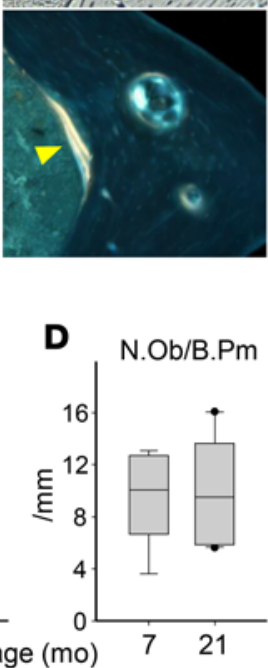

H
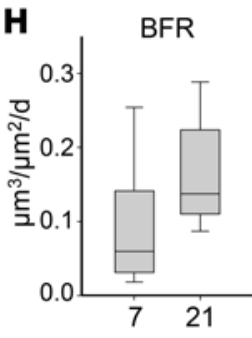

Figure 5. Endocortical remodeling becomes unbalanced in favor of resorption with advancing age. (A) Unstained cross-sections of the distal metaphysis (met) and the diaphysis (dia) with endosteal surface labeled by tetracycline (yellow arrowheads) given at 7 and 3 days before euthanasia of 6 - and 26-month-old female B6 mice. Original magnification: 40x. Right hand panels depict high power brightfield (upper) and fluorescent images of the area denoted by the box. (B) Toluidine blue-stained longitudinal sections with irregular scalloped cement lines (yellow arrowheads) in 7- and 21-month-old female B6 mice. Scale bar: $20 \mu \mathrm{m}$. (C-H) Histomorphometric determination of endosteal osteoclast number (N.Oc./B.Pm), osteoblast number (N.Ob/B.Pm), mineralizing surface (MS/BS), wall width (W.Wi), mineral apposition rate (MAR), and bone formation rate (BFR) in 7-monthold mice $(n=9)$ and 21-month-old $(n=10)$ mice. ${ }^{*} P<$ 0.05 vs. 7 -month-old animals by two-tailed $t$ test.

age (mo)

are incorporated into the bone when it is first formed by compaction and infilling of trabecular bone (37-39). Subsequent intracortical remodeling by osteoclasts and osteoblasts emanating from these vessels does occur, but only rarely.

Histologic examination of the femoral cortex of 21-month-old female B6 mice revealed the presence of both small and large pores (Figure 8, A-G). An increase in the number of large pores accounted for the increase in porosity (Figure $8, \mathrm{H}$ and I), and the magnitude of porosity was highly variable, in agreement with the microCT-based measurements. Cement lines formed boundaries around both small and large pores, defining packets of bone that contained osteocytes with canaliculi in contact with a central capillary (Figure 8, A, B, D, and E). Concentric lamellae were sometimes observed. Regardless of size, many pores were lined with osteoclasts (Figure $8 \mathrm{C}$ ), as well as osteoblasts, as indicated by the fluorochrome labeling present in an adjacent section (Figure 8, D and E). Intracortical labeling, as well as endosteal labeling, was often adjacent to scalloped cement lines (Figure 8, D and E). These histologic properties identify the cortical pores of aged female mice as osteons (9), many of which contain BMUs that are engaged in the remodeling of the interior of the femoral cortex. Osteons were present in bone that had not been previously remodeled (Figure 8D) and in parts of the cortex that had been remodeled, as indicated by the presence of irregular scalloped cement lines (Figure 8, A, E, and F). Pores were rarely observed in the periosteal portion of the cortex (Figure 8F), which was identified by the presence of parallel smooth cement lines, as we have previously described (25). Some pores were associated with capillaries originating in the BM (Figure $8 \mathrm{~F}$ ). Large pores contained cells with features that were indistinguishable from those of hematopoietic BM, as well as adipocytes (Figure 8G). There was no evidence of the dense fibrovascular stroma that characterizes lesions in cortical bone of aged 
A

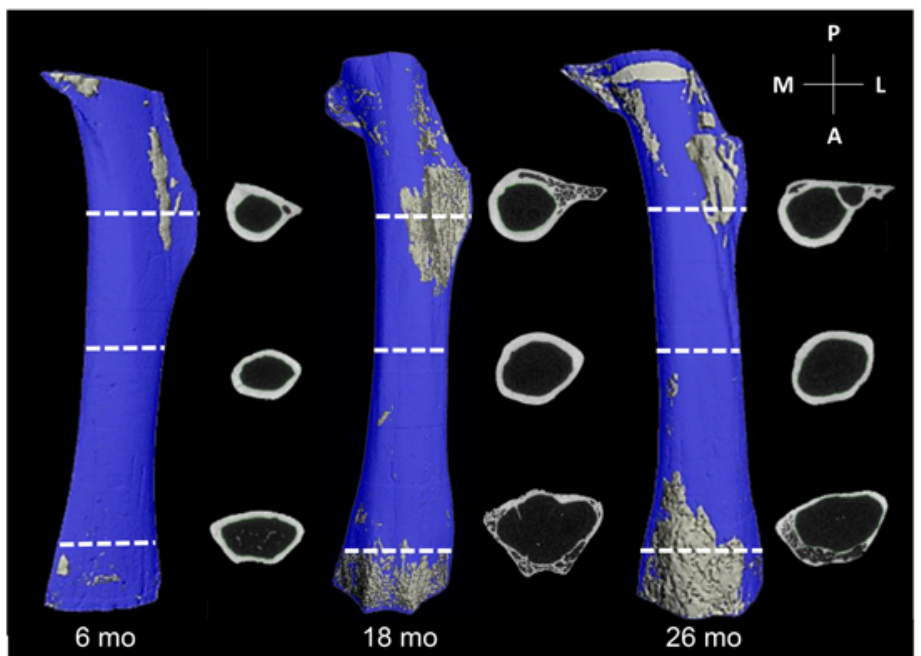

B

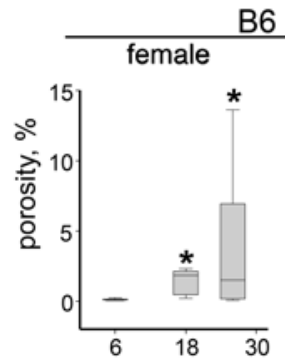

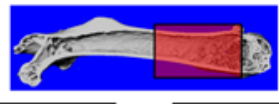

male

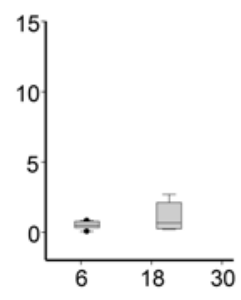

age (mo)

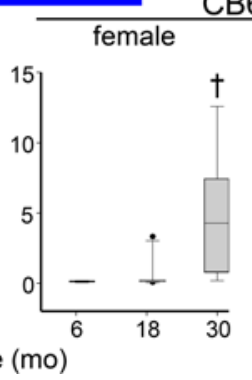

CB6F1

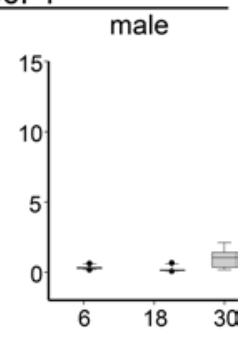

Figure 6. Femoral cortical porosity increases with age in females, but not males, in B6 and CB6F1 mice. (A) Inverse microCT images of femora (proximal at top) from female B6 mice, excluding epiphyses. Cortical voids are depicted in gray within a blue bone matrix. Lines show location of single microCT slices (before binarization). Orientation of slices: $P$, posterior; A, anterior; M, medial; L, lateral. (B) Quantification of porosity of the distal half of the femur, as indicated by the red box. Number of mice analyzed (ordered by increasing age): female B6 $(8,8,7)$; male B6 $(10,10)$; female CB6F1 $(8,10$, 10); male CB6F1 $(8,10,10) .{ }^{*} P<0.05$ vs. 6 -month B6 females; $\dagger P<0.05$ vs. 7 -month and 19 -month CB6F1 females, using a permutation approach to Student's two-tailed $t$ test.

mice of other strains $(40,41)$. Some aged male B6 mice also had large fluorochrome-labeled pores in the femoral cortex (Supplemental Figure 3, A-D), but most did not. As in the microCT findings of Figure $6 \mathrm{~B}$, porosity in males was highly variable, as determined by histomorphometry, and this prevented statistical demonstration of an age-dependent increase (Supplemental Figure 3E).

Cortical osteocytes of aged mice exhibit increased senescence and altered expression of proteins that affect bone remodeling. Changes in osteocyte function with age may contribute to age-related bone loss $(30,42,43)$. However, osteocyte age is not identical to chronologic age. To gain an appreciation of osteocyte age in adult mice prior to the onset of significant cortical bone loss, we analyzed the femoral cross sections of the 11-month-old female B6 shown in Figure 4. In these mice, bone of the interior cortex, and therefore the embedded osteocytes, that was made at 0.6 months (green) were still present in much of the proximal femur (Figure 4B), and in the medial region of the diaphysis (Figure 4C), but not in the distal metaphysis (Figure 4D). Osteocytes made at 1.4 months (red) and 2.6 months (yellow) were still present throughout the femur of these 11-month-old mice. This red and yellow labeling was mainly located in the outer half of the cortex, particularly in the distal metaphysis (Figure 4D). Most of the osteocytes located in the inner half of the cortex are lost by 18-26 months of age because of medullary expansion (Supplemental Tables 3 and 4). Based on these observations, we infer that the cortex of 18- to 26-month-old female B6 mice still contains osteocytes that were made during growth and are therefore much older than those in young-adult mice. Although new osteocytes are made during the slow periosteal apposition that continues throughout life, this bone comprises only $20 \%$ of the total cortex in 21-month-old mice (Table 1).

We next sought evidence for signs of increased osteocyte damage with age. Extracts of osteocyte-enriched femoral cortical bone from 21-month-old female B6 mice had higher levels of phospho-H2A histone family member $\mathrm{X}(\gamma-\mathrm{H} 2 \mathrm{AX})$, a marker of DNA double-strand breaks, as compared with extracts from 7-month-old mice (Figure 9A). Cumulative osteocyte death, as indicated by the presence of empty lacunae, in the endosteal region of the cortex was not affected by age (Table 1). However, osteocyte death in the periosteal portion of the cortex was lower than in the endosteal region. 
A

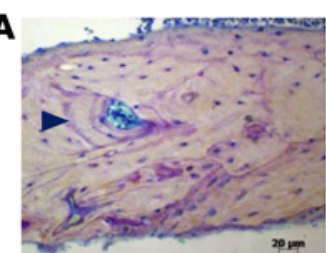

D

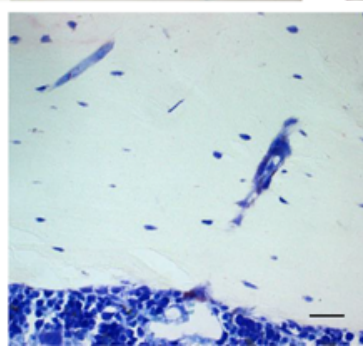

B
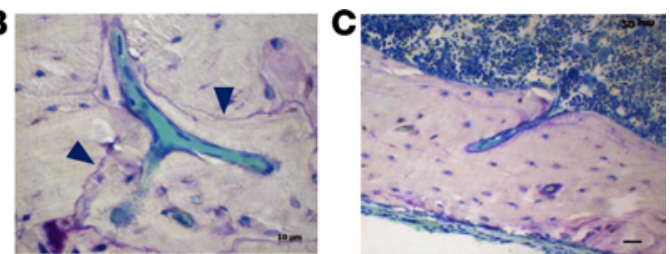

E

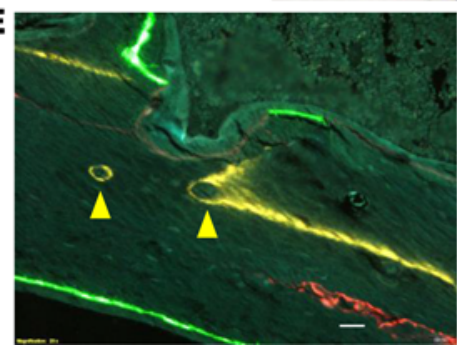

Figure 7. The femoral cortex of young-adult B6 female mice contains capillaries but does not exhibit intracortical remodeling. (A-D) Representative photomicrographs of pores in the femoral cortex of 7-month-old female B6 mice viewed in nondecalcified toluidine blue-stained (A-C) or TRAP-stained (D) longitudinal sections. Cement lines are marked by black arrowheads. (E) Yellow demeclocycline fluorescence labeling of small pores (yellow arrowheads) in a longitudinal section from an 11-month-old female B6 mouse given this fluorochrome at 2.6 months of age, as described in Figure 4. Scale bars: $20 \mu \mathrm{m}$ (C-E).

DNA damage is known to activate pathways that lead to cellular senescence, even in postmitotic cells (44). Compared with 7-month-old mice, femoral extracts from 21-month-old female B6 mice exhibited increased protein levels of the transcription factor GATA4 and decreased levels of the autophagosomal cargo protein p62 (Figure 9, A and B), both of which are senescence responses to DNA damage (45). In addition, transcript levels of the senescence marker p16 were elevated (Figure 9C). Furthermore, there was an age-dependent increase in the expression of stromal cell derived factor 1 (Sdf1, also known as Cxc112) and the metalloproteinase MMP-13 (Figure 9C), which are components of the senescence-associated secretory phenotype (SASP) (46). Femoral extracts from aged male B6 mice also had increased $\gamma$-H2AX levels, as well as increased GATA4, p16, and Mmp-13 expression (Figure 9, D-F), as compared with young adult controls; however, the levels of p62 and Sdf1 were not affected.

We also investigated the effect of aging on the expression of factors that control bone remodeling. Osteocyte-enriched bone preparations of aged female B6 mice exhibited an increase in RANKL transcripts (Figure 10A). BM plasma levels of soluble RANKL (sRANKL) were also increased, as compared with that of 7-month-old mice (Figure 10B). A similar age-dependent increase in sRANKL was observed in a second experiment (not shown). OPG transcripts, on the other hand, decreased with age, but the level of this protein in marrow plasma was unaffected (Figure 10, C and D). Interestingly, aging also caused a decrease in the expression of Wnt16 (Figure 10E) in females, which inhibits osteoclastogenesis via stimulation of OPG (47). Wnt5a, which promotes the differentiation of osteoclasts and osteoblasts $(48,49)$, was expressed at low levels and did not change with age (Figure 10F). Expression of the Wnt antagonist Sost, as well as BM plasma levels of sclerostin, declined with age (Figure 10, G and $\mathrm{H}$ ). However, this could not be accounted for by a decline in the number of cortical osteocytes (Table 1). Dkk1 transcripts were also reduced in cortices of aged mice, but protein levels in marrow plasma were unchanged (Figure 10, I and J). Wnt10b and Wnt 4 were the only other pro-osteoblastogenic Wnt ligands expressed at a detectable level, but only Wnt10b declined with age (Figure 10, K and L).

\section{Discussion}

The studies described herein demonstrate extensive loss of mass and structural deterioration of cortical bone in mice between 6-7 and 18-30 months of age. The cortical thickness of the femur decreased in both males and females, but the magnitude was greater in $\mathrm{B} 6$ than in $\mathrm{CB} 6 \mathrm{~F} 1$ mice. On the other hand, the age-dependent increase in cortical porosity was notable only in females. The trabecular bone mass of the vertebrae also decreased with age, but in contrast to cortical bone loss, trabecular bone loss was not affected by sex or genotype. In female B6 mice, loss of cortical bone was due to unbalanced BMU-based endosteal remodeling and de novo unbalanced osteonal remodeling.

The decrease in cortical thickness in femora of aged B6 mice occurred at both the diaphysis and metaphysis, but CB6F1 mouse cortical thinning only occurred at the metaphysis and at a later age. These observations are consistent with previous reports showing that genetic background greatly influences the gain or loss of murine cortical bone mass during growth, in old age, or following sex steroid deficiency (39, 

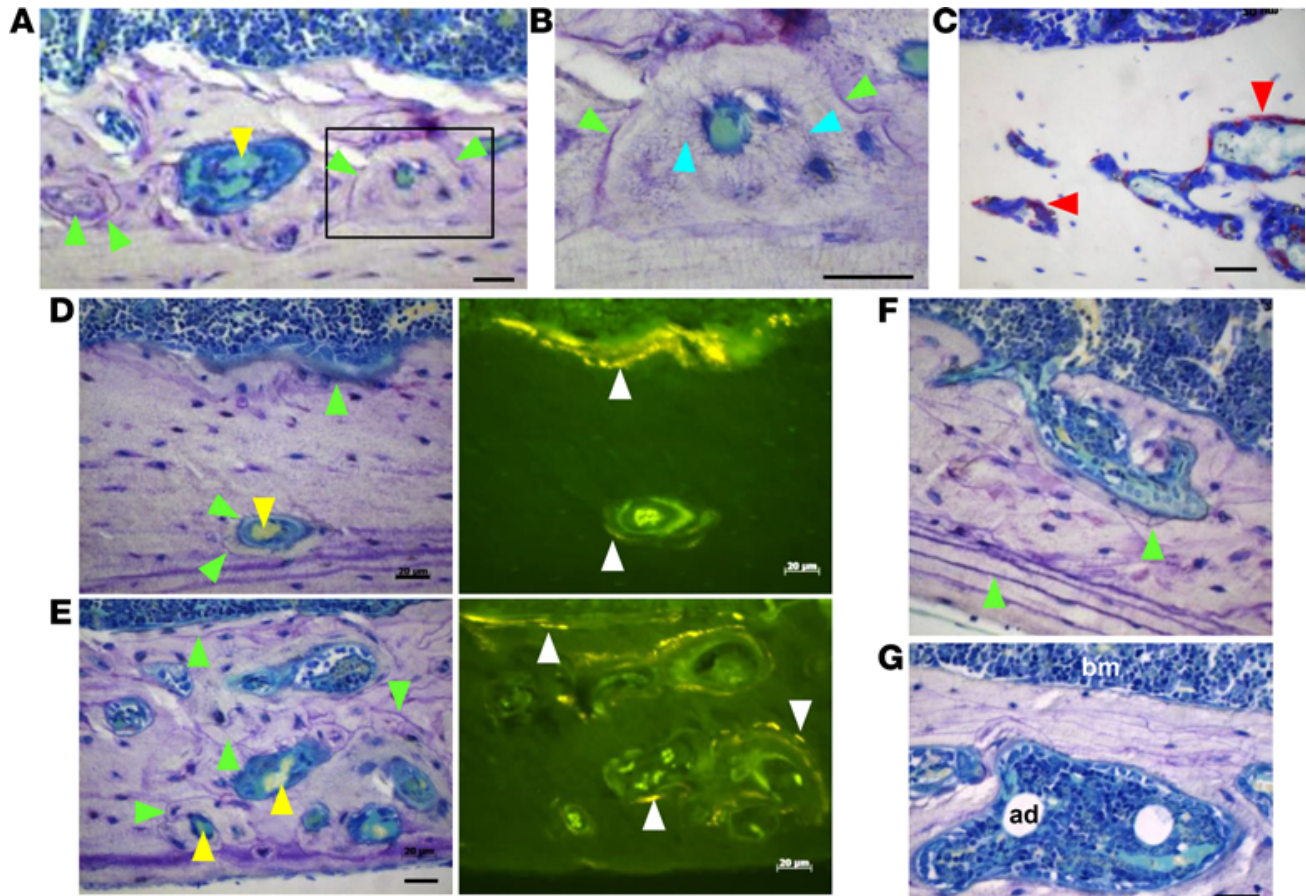

E
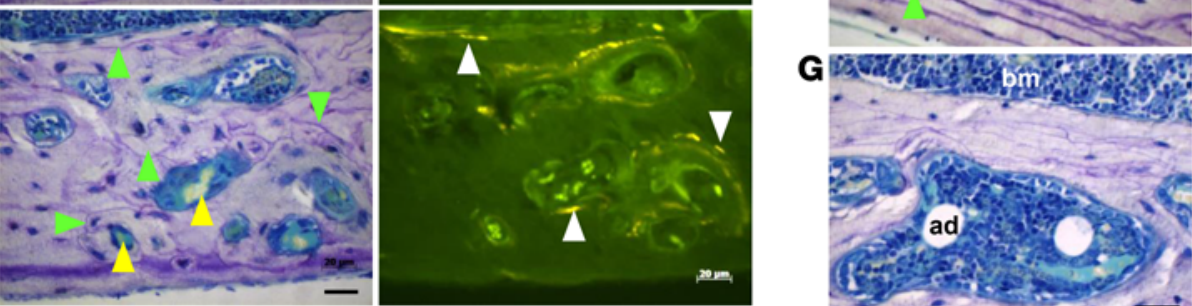

H

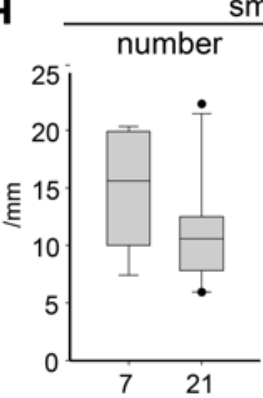
small voids

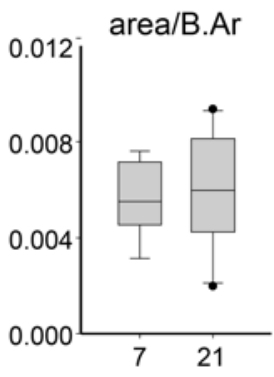

I

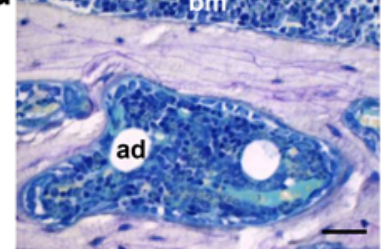

large voids
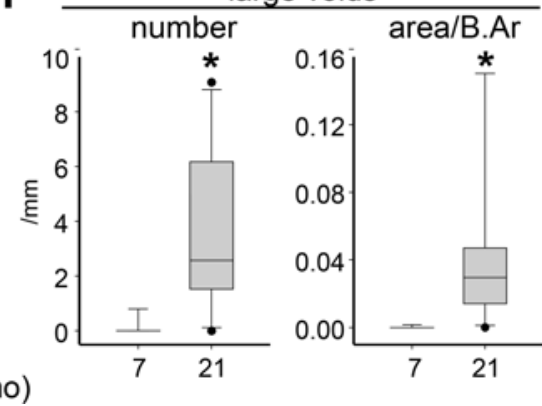

Figure 8. De novo osteonal remodeling in 21-month-old female B6 mice. (A-G) Representative photomicrographs of pores in the femoral cortex of 21-month-old female B6 mice viewed in nondecalcified toluidine blue-stained sections. The right portion of $\mathbf{D}$ and $\mathbf{E}$ show an unstained adjacent section under fluorescence illumination to visualize tetracycline (white arrowheads) given at 7 and 3 days before euthanasia. $\mathbf{B}$ is a high-power image of the area denoted by the box in A. Arrowheads mark red blood cells (yellow), cement lines (green), canalliculi (turquoise), and TRAP-positive osteoclasts (red); ad, adipocytes. Scale bars: $20 \mu \mathrm{m}$. (H and I) Histomorphometric determination of pore number and pore area per cortical bone area (B.Ar) in 7-month-old ( $n=$ 8) and 21-month-old $(n=10)$ female B6 mice. ${ }^{*} P<0.01$ vs. 7-month-old mice using a permutation approach to two-tailed Student's $t$ test.

50). Metaphyseal cortical bone loss with age was not detected in an earlier study of B6 mice (34). The most likely explanation for this discrepancy is that our measurements were limited to the middle third of the metaphysis, as opposed to the entire metaphysis in the earlier study. We deliberately measured this region because cortical thickness varies considerably in the proximal vs. the distal part of the metaphysis. This makes it more difficult to detect an age-dependent change in the thickness of this part of the cortex when averaged over the entire metaphysis.

Importantly, our finding that OPG ablated endosteal bone formation establishes that the endosteal surface of adult mice undergoes BMU-based remodeling, not osteoclastic modeling. The same is evidently true in adult rats, as shown by histologic analysis of 12-month-old female Fischer-344 rats (36) and by the strong inhibitory effect of the antiresorptive drug risedronate on endosteal fluorochrome labeling in 15-month-old ovariectomized Sprague-Dawley rats (51). The demonstration of scalloped cement lines on the endosteal surface of both 7- and 21-month-old B6 mice in the present study, as well as the association of these cement lines with fluorochrome labeling in aged mice, shows that BMU-based remodeling of the endosteal surface persists with advancing age. Therefore, as is the case in humans $(15,16)$, age-dependent cortical thinning in mice is due to loss of bone from the endosteal surface that exceeds 

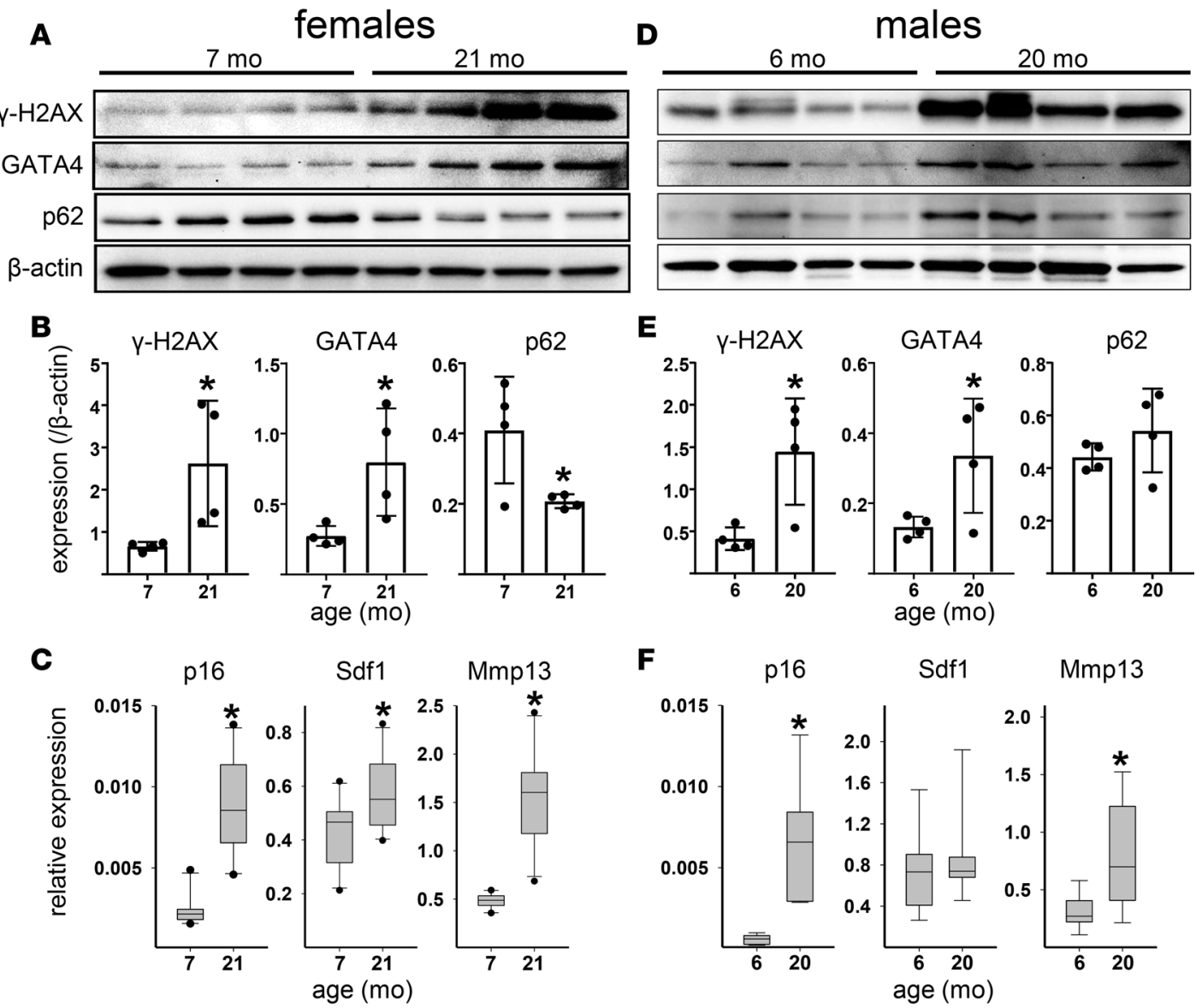

Figure 9. Osteocyte-enriched bone from aged mice exhibits markers of cellular senescence. Western blot analysis and quantification of markers of DNA damage $(\gamma-\mathrm{H} 2 \mathrm{AX}$ ) and cellular senescence (GATA4 and p62) in femoral extracts from female (A and B) or male ( $\mathbf{D}$ and $\mathbf{E})$ B6 mice. Transcript levels of the senescence marker p16 ink4a (p16), and the SASP proteins Sdf1 and Mmp-13 were determined by qPCR of extracts from 7-month-old $(n=9)$ and 21-month-old $(n=10)$ female B6 mice (C), or from 6-month-old $(n=9)$ and 20 -month-old $(n=9)$ male B6 mice $(\mathbf{F}) .{ }^{*} P<0.05$ vs. 7-month-old sex-matched animals by two-tailed Student's t test, or by Rank Sum test, after adjustment of $P$ values for repeated measurements by the Benjaminji-Hochsberg method.

the amount of bone added to the periosteal surface. This effect of aging results from inadequate filling of resorption cavities due to an increase in osteoclasts and decrease in osteoblasts, documented here and elsewhere $(23,52)$. We had previously shown that insufficient osteoblasts accounts for the unbalanced remodeling and, thereby, the age-dependent loss of trabecular bone in vertebrae of B6 mice. Importantly, however, osteoclast number declines with age in this compartment (22). Taken together with the evidence of the present report that genetic background influences age-dependent cortical thinning but not trabecular bone loss, we conclude that distinct molecular mechanisms underlie the age-dependent dysregulation of endosteal and trabecular remodeling in mice.

The present evidence that de novo unbalanced intracortical remodeling accounts for the age-dependent increase in cortical porosity in mice notwithstanding, some aspects of the biology of osteons in aged mice and humans are different. Cortical bone is initially formed by infilling of trabecular spicules under the periphery of the growth plate, which also entraps capillaries (37-39). In humans and other large mammals, the perivascular space between the vessels and bone is filled in with layers of concentric lamellae to form a primary osteon. Secondary osteons subsequently form by extravasation of osteoclasts and delivery of osteoblasts from the capillaries during growth and remodeling of the cortex. Our findings in young-adult mice show that cortical capillaries formed during development persist in the cortex, in agreement with synchrotron-based imaging studies (53). Osteons arise only occasionally from these capillaries in mice, as evidenced by low levels of fluorochrome-labeled pores in young-adult mice. With advancing age, however, osteons do form in mice, and they exhibit histologic hallmarks of remodeling activity, including osteoclasts and fluorochrome-labeled bone matrix adjacent to scalloped cement lines. However, murine osteons 

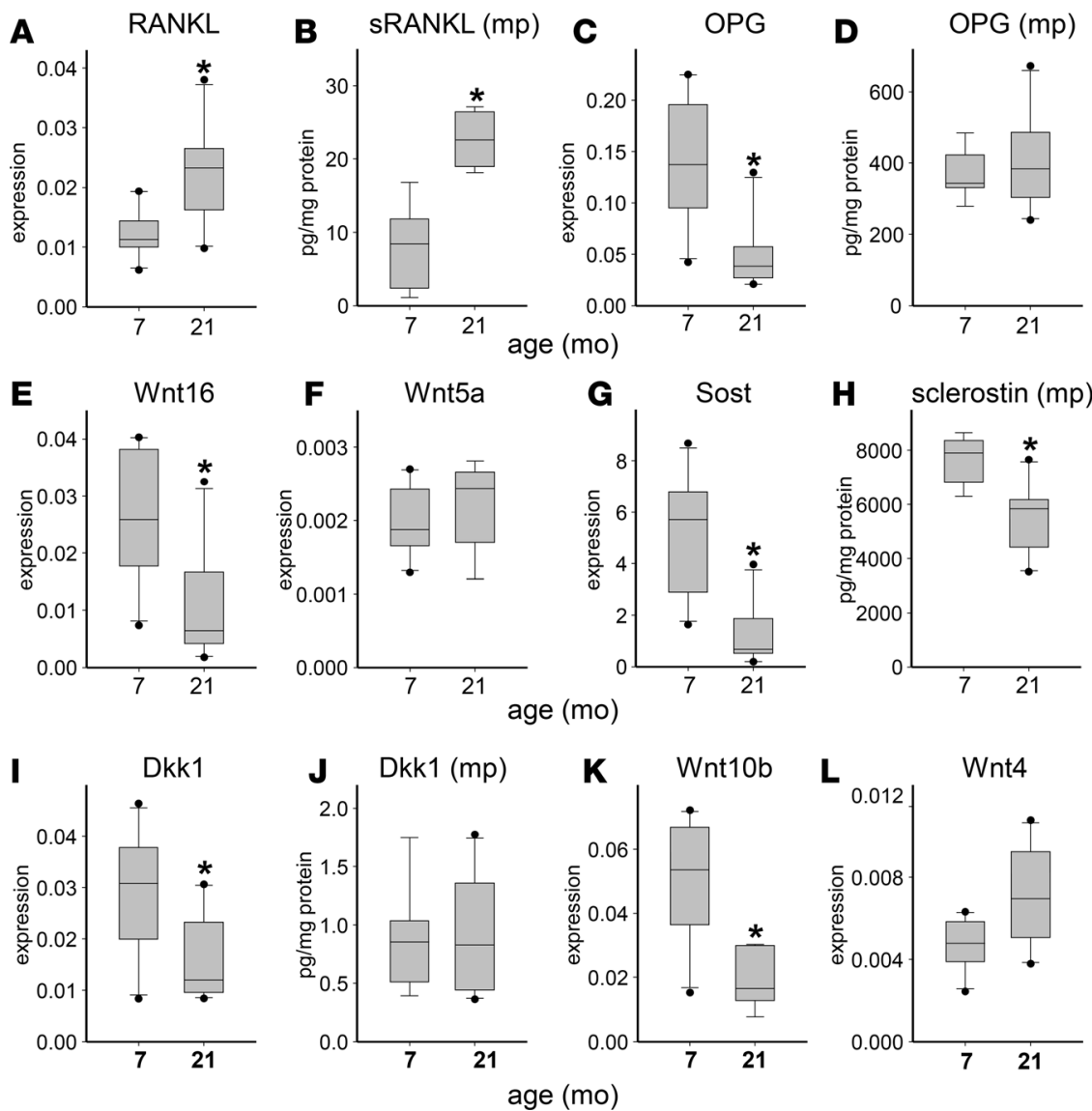

Figure 10. Osteocyte-enriched bone from aged mice exhibits changes in the expression of factors involved in the regulation of osteoclast and osteoblast differentiation. (A-L) Transcript levels and femoral marrow plasma ( $\mathrm{mp}$ ) protein levels of indicated genes were determined by qPCR or ELISA, respectively, using extracts from the 7-month-old $(n=9)$ and 21-month-old $(n=10)$ female B6 mice of described in Figure $9 .{ }^{*} P<0.05$ vs. 7-month-old mice by two-tailed $t$ test or Rank Sum test. $P$ values for the effect of age on transcript levels were adjusted for repeated measurements by the Benjaminji-Hochsberg method.

generally lack the numerous concentric lamellae that characterize the much larger osteons of human bone and, instead, resemble rabbit osteons (54). Moreover, murine osteons do not exhibit the highly organized networks seen in humans, perhaps reflecting the lack of a preexisting Haversian system.

In aged female mice, large pores are especially prominent in the metaphyseal cortex, and they probably arise from a merger of nearby osteons. As in aged humans, unbalanced osteonal remodeling is most evident near the endosteal surface. This leads to the penetration of the endosteal boundary and the trabecularization of the endocortical part of the cortex, similar to the situation in humans. The present histologic and microCT imaging studies suggest that, in aged mice, new osteons may arise from preexisting cortical capillaries, from redirection of endosteal BMUs from the endosteal surface into the cortex in tandem with vascular invasion from the marrow, or from both mechanisms. Similar detailed histologic and microCT imaging studies have not yet been done in cortical bone of aged humans, but the connectivity of vascular channels in humans increases with age (11). It is therefore tempting to speculate that, analogous to the situation in mice, age-dependent formation of new osteons by unbalanced BMUs contributes to the development of cortical porosity in humans.

Damage to long-lived cells, together with the cellular response to damage, are thought to be involved in the functional deterioration of many tissues with advancing age (55). We show here that osteocytes formed during growth are still present in the cortical bone of skeletally mature and of aged mice. Though not based on direct measurements, mean bone age, and therefore osteocyte age, doubles between age 30 and 70 in human ribs (56). Therefore, relative to chronologic age, cortical osteocytes of aged mice are probably older than those in aged humans because of the lack of intracortical remodeling until the latter half of the murine lifespan.

Importantly, we found that increased osteocyte age was associated with an increase in markers of DNA damage, cellular senescence, and SASP in osteocyte-enriched cortical bone preparations from both male and female B6 mice, consistent with recent findings by others (57). The SASP may well influence the secretory profile of neighboring aged but healthy osteocytes, as well as the relatively young osteocytes formed during endosteal and intracortical remodeling (58). Indeed, the studies in female mice shown herein revealed an age-dependent increase in RANKL, as well as a decrease in OPG that should potentiate 
the stimulatory effect of RANKL on the genesis and survival of osteoclasts. The decrease in OPG could be due to decreased Wnt16 expression $(47,59)$, which we also documented here. Expression of the SASP cytokines Sdf1 and MMP-13 (60) also increased with aging. Both of these cytokines enhance RANKLinduced osteoclastogenesis $(23,61)$. Osteocyte-derived RANKL plays an important role in the homeostasis of cortical bone, as evidenced by the fact that conditional deletion of this cytokine in osteocytes increases cortical thickness in young-adult mice and attenuates the cortical thinning caused by estrogen deficiency or glucocorticoid excess $(62,63)$. However, we cannot exclude the possibility that other cells also contribute to the increased sRANKL in marrow of aged murine bone.

In contrast to our findings, others have reported that RANKL and OPG transcripts in murine cortical bone are unaffected by age (20). Technical differences may explain this discrepancy, since - in contrast to the earlier study - we did not use GAPDH as one of the gene normalizers because its expression did not correlate with any of the 4 other housekeeping genes. We cannot explain why we observed increased sRANKL in BM plasma with age, while the earlier study, using a similar immunoassay, reported a decrease in sRANKL in the BM (20).

The decrease in Sost gene expression and sclerostin protein in marrow plasma reported herein constitutes additional evidence that aging alters the secretome of cortical osteocytes and is consistent with previous studies $(20,64)$. Decreased sclerostin levels should increase pro-osteoblastogenic Wnt signaling (65), but we have shown previously that expression of Wnt target genes declines in murine cortical bone with age (66, 67). This could be due to a decline in Wnt ligands like Wnt10b, as reported here, and to ROS-mediated activation of FoxO transcription factors, leading to a reduction in the $\beta$-catenin required for Wnt signaling (42, 68). The functional role of ROS in age-dependent bone loss has been demonstrated by attenuation of the age-related decline in femoral cortical thickness by overexpression of catalase in cells of the osteoblast lineage (23). Finally, other studies of ours suggest that senescence of osteoblast progenitors leading to cell cycle arrest increases with age, which may contribute to the reduction in the number of endosteal osteoblasts (69).

Earlier work had suggested that increased osteocyte apoptosis, perhaps in response to age-dependent bone fatigue and microdamage, contributed to the development of cortical porosity $(30,70,71)$. This notion was based on evidence that apoptotic osteocytes released factors that stimulated RANKL production by neighboring viable osteocytes. Moreover, we had reported that the age-dependent increase in RANKL and cortical porosity are exacerbated in female mice with apoptosis-resistant osteocytes (25), probably due to accumulation of damaged osteocytes that cannot complete the apoptotic death program. The findings of the present report raise the possibility that, besides apoptotic osteocytes, senescent osteocytes are involved in the activation of de novo intracortical remodeling via SASP. Nonetheless, the highly variable magnitude and preferential development of cortical porosity near the metaphyses remains unexplained. Similarly, we have no explanation for the much smaller magnitude of porosity in male compared with female mice in the face of a seemingly equivalent increase in osteocyte senescence in both sexes. Future studies involving pharmacologic or genetic manipulation of osteocyte senescence may shed light on these issues.

Similar to our findings in mice, HRpQCT measurements of the radius and tibia show that postmenopausal women have greater cortical porosity than men over the age of $50(5,6)$. It is unknown whether this difference is due to decreased estrogens at the menopause. Be that as it may, the studies reported herein show that male and female mice exhibit an equivalent age-dependent decline in vertebral trabecular bone and femoral cortical thickness - changes known to be influenced by sex steroid deficiency (23). Therefore, a greater decrease in sex steroid levels in females than in males with age cannot account for the greater porosity of cortical bone in female mice. One possibility is that the higher level of androgens in male mice and humans protects against the development of cortical porosity. Alternatively, the female skeleton may be genetically more sensitive to the development of age-dependent porosity than the male skeleton, analogous to the influence of sex-specific loci on BMD in children (72).

In summary, the evidence presented in this report shows that the femoral cortical bone of mice undergoes BMU-based remodeling. Endosteal remodeling becomes progressively unbalanced in favor of resorption in both sexes following skeletal maturation, and unbalanced osteonal remodeling starts de novo with advancing age, particularly in females. These events may be caused by an altered secretome of cortical osteocytes, which could be due in part to senescence. The cumulative result is a thin, trabecularized, and porous cortex. Based on this evidence, we conclude that the cellular and molecular mechanisms underlying the age-dependent cortical bone deterioration in mice are indeed relevant and informative to the pathologic mechanisms responsible for the decreased thickness and increased porosity of cortical bone that occurs with advancing age in humans. 


\section{Methods}

Mice. Male and female B6 and CB6F1 mice of specific ages were obtained from NIH-supported colonies maintained by Charles River Laboratories (73) and fed NIH-41 Rat and Mouse Diet/5F (TestDiet, catalog 54709) containing $18 \%$ protein and $4.7 \%$ fat. After receipt at the University of Arkansas for Medical Sciences, mice were maintained at a constant temperature of $23^{\circ} \mathrm{C}$ and a 12 -hour light/dark cycle, and they were maintained on Teklab global 14\% protein rodent maintenance diet (Envigo, catalog 2014) containing $14 \%$ protein and $4 \%$ fat and acidified water ad libitum. Within 3 weeks after receipt from Charles River Laboratories, mice were injected with tetracycline $(15 \mathrm{mg} / \mathrm{kg}$ body weight $)$ at 7 and 3 days before euthanasia (by $\mathrm{CO}_{2}$ inhalation) to allow quantification of bone formation.

The effect of OPG on endosteal bone formation was determined using femora obtained in a previously published study (74) that focused on vertebral trabecular bone. Briefly, female Swiss-Webster mice (6 months old, 29-31 g) were obtained from Harlan and fed a standard rodent diet (Teklad 22/5, catalog 8640). Mice were injected with PBS (vehicle) or $10 \mathrm{mg} / \mathrm{g}$ of human OPG-Fc (provided by Paul Kostenuik, Amgen, Seattle, Washington, USA) on day 0, 7 and 14, and they were euthanized on day 19. Tetracycline (30 mg/kg, i.p.) was given 7 days and alizarin red (30 mg/kg, i.p.) 2 days before euthanasia to mark sites of bone formation.

Multiple fluorochrome labeling was performed at Indiana University with 2 female B6 mice, raised from birth from breeders obtained from the Jackson Laboratory, maintained on standard mouse chow (Harlan Teklad 2018SX; 1\% Ca; 0.65\% P; vitamin D3 [2.1 IU/g]) and acidified water ad libitum. Mice were injected (i.p.) with bone-labeling fluorochromes as follows: $100 \mu \mathrm{l}$ of $0.1 \%$ calcein at 18 days $(0.6$ months) of age; $150 \mu \mathrm{l}$ of $0.18 \%$ alizarin complexone at 6 weeks ( 1.4 months) of age; $200 \mu 1$ of $0.6 \%$ demeclocycline at 11.1 weeks (2.6 months) of age; $200 \mu 1$ of $0.1 \%$ calcein at 41 weeks (9.5 months) of age; and $200 \mu \mathrm{l}$ of $0.18 \%$ alizarin complexone at 46 weeks (10.7 months) of age. Mice were euthanized at 47.1 weeks (11.0 months) of age.

Bone imaging. BMD of the left femur, lumbar spine, and whole body (excluding the head) was determined in sedated mice ( $2 \%$ isoflurane) by dual-energy X-ray absorptiometry using a PIXImus densitometer (GE Lunar) (75). Bone architecture was determined on dissected femora and lumbar vertebra (L4) cleaned of adherent tissue, fixed in Millonig's phosphate buffer (Leica Microsystems), and stored in 100\% ethanol. Bones were scanned by microCT with a MicroCT40 (Scanco Medical) at high resolution ( $6 \mu \mathrm{m}$ isotropic voxel size) to obtain images and at medium resolution $(12 \mu \mathrm{m}$ isotropic voxel size) for quantitative determinations. For the latter, a Gaussian filter (sigma $=0.8$, support $=1$ ) was applied.

Scanco Eval Program v.6.0 was used for measuring bone volume. Scan settings included X-ray tube potential $(55 \mathrm{kVp})$, X-ray intensity $(145 \mu \mathrm{A})$, and integration time $(220 \mathrm{~ms})$. Nomenclature conforms to recommendations of the American Society for Bone and Mineral Research (76). Femora were scanned from a point immediately distal to the third trochanter to the beginning of the distal growth plate. Cortical dimensions were determined at the diaphysis (18 slices, midpoint of the bone length as determined in scout view) and metaphysis, starting $3.6 \mathrm{~mm}$ (300 slices) from the diaphysis and proceeding distally for 50 slices, to obtain cross-sectional images drawn to exclude trabecular elements. Cortical analysis were measured at a threshold of $260 \mathrm{mg} / \mathrm{cm}^{3}$. Trabecular bone measurements at the distal femur were made on 151 slices beginning 8-10 slices away from the growth plate, so as to avoid the primary spongiosa, and proceeding proximally. Trabecular analyses were performed on contours of the cross-sectional images drawn to exclude cortical bone and were measured at a threshold of $220 \mathrm{mg} / \mathrm{cm}^{3}$ and using sphere filling distancetransformation indices without assumptions about the bone shape as a rod or plate.

For femoral porosity measurements, slices were analyzed from a point immediately distal to the third trochanter to a point immediately adjacent to the primary spongiosa. After defining endosteal and periosteal boundaries, an additional image processing script ("peel-iter = 2") was used to eliminate false voids caused by imperfect wrap of the contours to the bone surface. Images were binarized with a threshold of $365 \mathrm{mg} / \mathrm{cm}^{3}$. Cortical bone volume and void volume were determined with the "cl_image" script and used to calculate porosity. To avoid inclusion of osteocyte lacunae and canalicular space, void volumes $<31,104$ $\mu \mathrm{m}^{3}$ (18 voxels) were excluded in the determination of porosity.

The fourth lumbar vertebra (L4) was scanned from the rostral growth plate to the caudal growth plate to obtain 233 slices. BV/TV in the vertebra was determined using 100 slices $(1.2 \mathrm{~mm}$ ) of the anterior (ventral) vertebral body immediately inferior (caudal) to the superior (cranial) growth plate. Trabecular bone analyses were performed on contours of cross-sectional images, drawn to exclude cortical bone, as described for femoral 
trabecular bone. Cortical bone thickness was determined on the ventral cortical wall using contours of crosssectional images, drawn to exclude trabecular bone, as described for femoral cortical bone.

Histology. Femora were fixed in Millonig's and embedded nondecalcified in methyl methacrylate. For histomorphometric measurements, $5 \mu \mathrm{m}$-thick longitudinal sections were cut in the medial-lateral plane. For histologic characterization, additional sections were cut at approximately $25 \%, 50 \%$, and $75 \%$ of the distance from the top of the bone. For preparation of cross sections, blocks were marked at the midsection for diaphyseal sections and $1 \mathrm{~mm}$ below the distal growth plate for metaphyseal sections. Bones were then cut with a Buehler IsoMet low-speed saw to prepare a 200-300 $\mu \mathrm{m}$ section at each site and were ground to a final thickness of $\sim 100 \mu \mathrm{m}$. For studies on the effect of OPG on endosteal bone formation, midlongitudinal sections were made for TRAP staining. The remnants were then deplasticized and reoriented, and finally reembedded in plastic for preparation of cross sections.

Nondecalcified sections were stained for tartrate-resistant acid phosphatase to visualize osteoclasts using napthol AS-MX and Fast Red TR salt (Sigma-Aldrich). Sections were also stained with 0.3\% toluidine blue (Sigma-Aldrich) in phosphate buffered citrate, $\mathrm{pH} \mathrm{3.7,} \mathrm{to} \mathrm{visualize} \mathrm{osteoblasts,} \mathrm{osteoid,} \mathrm{and}$ cement lines (77). The lamellar bone of the outer cortex was identified by the presence of parallel smooth cement lines (25). Unstained sections were used to quantify dynamic indexes of bone formation based on tetracycline labeling - specifically, total perimeter (B.Pm), single label perimeter (sL.Pm), double label perimeter (dL.Pm), and mineral apposition rate (MAR). These values were used to calculate mineralizing surface $(\mathrm{MS} / \mathrm{BS}=[1 / 2 \mathrm{sL} . \mathrm{Pm}+\mathrm{dL} . \mathrm{Pm}] / \mathrm{B} . \mathrm{Pm} \times 100 ; \%)$ and bone formation rate $(\mathrm{BFR} / \mathrm{BS}$ $=\mathrm{MAR} \times \mathrm{MS} / \mathrm{BS} ; \mu \mathrm{m} 2 / \mu \mathrm{m} /$ day) . Histomorphometric measurements were made in a blinded fashion using Osteomeasure (vXP 3.1, Osteometrics) on both of the endosteal surfaces of a longitudinal section. If only a single label was present, data were treated as missing values for statistical purposes. Histomorphometric parameters are reported using the nomenclature recommended by the American Society for Bone and Mineral Research (78).

For experiments involving multiple fluorochrome labeling during growth and adulthood, serial cross-sections were made from whole plastic-embedded femora that had been scanned in a Scanco $\mu$ CT35 at $10 \mu \mathrm{m}$ resolution. The microCT scans were used to align the serial histological sections to one another on a slice-by-slice basis. The blocks were then serially sectioned in the transverse plane using a low-speed saw. Each wafer was $\sim 120-\mu \mathrm{m}$ thick, and the kerf removed by each pass of the wafering blade was $\sim 180 \mu \mathrm{m}$, which puts each wafer $\sim 300 \mu \mathrm{m}$ apart. Each wafer was ground to a final thickness of $\sim 30 \mu \mathrm{m}$.

Western blotting. Osteocyte-enriched femoral cortical bone was prepared by removing the ends, flushing the BM by centrifugation, and removing surface cells by scraping with a scalpel. Bone fragments were immediately frozen in liquid nitrogen and pulverized. Proteins were extracted with a buffer containing 20 $\mathrm{mM}$ Tris-HCL, $150 \mathrm{mM} \mathrm{NaCl}, 1 \%$ Triton X-100, protease inhibitor mixture, and phosphatase inhibitor cocktail (Sigma-Aldrich) on ice for 30 minutes and then kept at $-80^{\circ} \mathrm{C}$ for overnight. The protein concentration of the bone extract was determined using the DC Protein Assay Kit (Bio-Rad). The extracted protein (20-30 $\mu$ g per sample) was subjected to $8 \%$ or $15 \%$ SDS-PAGE gels and transferred electrophoretically onto PVDF membranes. The membranes were blocked in 5\% fat-free milk/Tris-buffered saline for 120 minutes and incubated with each primary antibody, followed by secondary antibodies conjugated with horseradish peroxidase. Mouse monoclonal antibodies against $\gamma-\mathrm{H} 2 \mathrm{AX}$ (Millipore, catalog 05-636, 1:5,000), rabbit polyclonal antibody for p62 (Sigma-Alrich, catalog P0067, 1:1,000), and goat polyclonal antibody for GATA4 (Santa Cruz Biotechnology, catalog sc-1237, 1:500) were used to detect their corresponding protein levels. Blots were stripped and reprobed with anti- $\beta$-actin antibody (Santa Cruz Biotechnology, catalog sc-81178, 1:2,000). Bound antibodies were detected with ECL reagents (Millipore) and were imaged and quantified with a VersaDocTM imaging system (Bio-Rad). Uncut gels are shown in Supplemental Figure 4.

Quantitative PCR. Osteocyte-enriched bone preparations were made as described above. Total RNA was extracted with Ultraspec (Biotecx Laboratories) and reverse-transcribed using the High-Capacity cDNA Archive Kit (Applied Biosystems) according to the manufacturer's instructions. Transcript levels were determined by Taqman quantitative PCR using primers, and probes were obtained from Applied Biosystems, as listed in Supplemental Table 7, using an ABI 7300 Prism instrument. Transcripts were normalized to each of 5 housekeeping genes (ChoB, ActB, B2m, 18S, and Hrpt1), and the transcript level were calculated as the geometric mean of the 5 normalized values. Pearson correlation coefficients between pairs of these referents ranged from $0.79-0.95$ ( $P<1 \times 10^{-6}$ for all comparisons). GAPDH was not used because of its poor correlation with other house-keeping genes $(\mathrm{r}=0.17-0.17, P=0.5-0.8)$. 
Statistics. Graphical data are shown as box-plots representing the $25^{\text {th }}-75^{\text {th }}$ percentile values. The line in the box denotes the median value. Whiskers represent the $95 \% \mathrm{CI}$, and dots represent data falling outside this CI. Graphs depicting Western blot quantification are presented as dot plots, along with bar graphs depicting the mean and standard deviation. Numerical tabular data presented in Supplemental Material is shown as the mean and SD. Data were analyzed by Student's two-tailed $t$ test or ANOVA, as appropriate, using Sigmaplot (version 12.5, Systat Software Inc.) or SAS (version 904, SAS Institute Inc.). If the normality or equal variance assumptions for standard parametric analysis methods were not met, data were analyzed by the Wilcoxon Rank Sum test. When ANOVA indicated a significant effect, pairwise multiple comparisons were performed and the $P$ values adjusted using the Holm-Sidak method. When pairwise repeated comparisons of the effect of age on transcript levels were performed, $P$ values were adjusted using the Benjamini-Hochbergmethod. In the case of porosity values, a permutation approach was used (79), in which a standard analysis is performed on the data and the factor labels are then randomized while keeping the observed values of the dependent variable of interest as is. The analysis is then recalculated and the process is repeated to obtain a sampling distribution of the statistics of interest. The $P$ values are then calculated from the sampling distribution. For all tests, $P<0.05$ was considered statistically significant

Study approval. Animal use protocols were approved by the IACUCs of the University of Arkansas for Medical Sciences, the Central Arkansas Veterans Healthcare System, and the Indiana University School of Medicine.

\section{Author contributions}

MA, AGR, SCM, CAO, and RLJ conceived and designed the experiments; MP, HNK, JX, MA, AGR, CAO, and RLJ generated and analyzed the data; JDT assisted in the performed statistical analyses; RLJ prepared the manuscript with the assistance of MP, MA, AGR, RSW, SCM, and CAO.

\section{Acknowledgments}

We thank Aubrey Hough, professor of anatomy, UAMS, for advice and inspection of bone sections for signs of lesions, and we thank Annick Deloose, Michela Palmieri, Priscilla Baltz, Stu Berryhill, and Julie Crawford for technical assistance. This work was supported by Biomedical Laboratory Research and Development Service of the Veterans Administration Office of Research and Development (I01 BX001478 to AGR, I01 BX000514 to RLJ, I01-BX000294 to CAO); the NIH (P01 AG-13918 to SCM, R01 AR56679 to MA, AR53237 to AGR, AR49794 to CAO); and the University of Arkansas for Medical Sciences Tobacco Funds and Translational Research Institute (1UL1 RR-029884).

Address correspondence to: Robert L. Jilka, University of Arkansas for Medical Sciences, 4301 W. Markham, Slot 587, Little Rock, Arkansas 72211, USA. Phone: 501.686.5130; Email: rljilka@uams.edu.

1. Roux C, et al. Burden of non-hip, non-vertebral fractures on quality of life in postmenopausal women: the Global Longitudinal study of Osteoporosis in Women (GLOW). Osteoporos Int. 2012;23(12):2863-2871.

2. Zebaze RM, et al. Intracortical remodelling and porosity in the distal radius and post-mortem femurs of women: a cross-sectional study. Lancet. 2010;375(9727):1729-1736.

3. Nicks KM, Amin S, Atkinson EJ, Riggs BL, Melton LJ, Khosla S. Relationship of age to bone microstructure independent of areal bone mineral density. J Bone Miner Res. 2012;27(3):637-644.

4. Vandenput L, et al. Serum estradiol levels are inversely associated with cortical porosity in older men. J Clin Endocrinol Metab. 2014;99(7):E1322-E1326.

5. Nirody JA, et al. Spatial distribution of intracortical porosity varies across age and sex. Bone. 2015;75:88-95.

6. Shanbhogue VV, Brixen K, Hansen S. Age- and Sex-Related Changes in Bone Microarchitecture and Estimated Strength: A Three-Year Prospective Study Using HRpQCT. J Bone Miner Res. 2016;31(8):1541-1549.

7. Burghardt AJ, Kazakia GJ, Ramachandran S, Link TM, Majumdar S. Age- and gender-related differences in the geometric properties and biomechanical significance of intracortical porosity in the distal radius and tibia. J Bone Miner Res. 2010;25(5):983-993

8. Bala Y, et al. Trabecular and cortical microstructure and fragility of the distal radius in women. J Bone Miner Res. 2015;30(4):621-629

9. Parfitt AM. Osteonal and hemi-osteonal remodeling: the spatial and temporal framework for signal traffic in adult human bone. J Cell Biochem. 1994;55(3):273-286.

10. Seeman E. Age- and menopause-related bone loss compromise cortical and trabecular microstructure. J Gerontol A Biol Sci Med Sci. 2013;68(10):1218-1225.

11. Cooper DM, Thomas CD, Clement JG, Turinsky AL, Sensen CW, Hallgrímsson B. Age-dependent change in the 3D structure 
of cortical porosity at the human femoral midshaft. Bone. 2007;40(4):957-965.

12. Bell KL, Loveridge N, Jordan GR, Power J, Constant CR, Reeve J. A novel mechanism for induction of increased cortical porosity in cases of intracapsular hip fracture. Bone. 2000;27(2):297-304

13. Reeve J, Loveridge N. The fragile elderly hip: mechanisms associated with age-related loss of strength and toughness. Bone. 2014;61:138-148.

14. Cooper DM, Thomas CD, Clement JG, Hallgrímsson B. Three-dimensional microcomputed tomography imaging of basic multicellular unit-related resorption spaces in human cortical bone. Anat Rec A Discov Mol Cell Evol Biol. 2006;288(7):806-816.

15. Han ZH, Palnitkar S, Rao DS, Nelson D, Parfitt AM. Effects of ethnicity and age or menopause on the remodeling and turnover of iliac bone: implications for mechanisms of bone loss. J Bone Miner Res. 1997;12(4):498-508.

16. Power J, Loveridge N, Lyon A, Rushton N, Parker M, Reeve J. Bone remodeling at the endocortical surface of the human femoral neck: a mechanism for regional cortical thinning in cases of hip fracture. J Bone Miner Res. 2003;18(10):1775-1780.

17. Almeida M, et al. Estrogens and Androgens in Skeletal Physiology and Pathophysiology. Physiol Rev. 2017;97(1):135-187.

18. Dutta S, Sengupta P. Men and mice: Relating their ages. Life Sci. 2016;152:244-248.

19. Halloran BP, Ferguson VL, Simske SJ, Burghardt A, Venton LL, Majumdar S. Changes in bone structure and mass with advancing age in the male C57BL/6J mouse. J Bone Miner Res. 2002;17(6):1044-1050.

20. Shahnazari M, et al. Bone turnover markers in peripheral blood and marrow plasma reflect trabecular bone loss but not endocortical expansion in aging mice. Bone. 2012;50(3):628-637.

21. Ferguson VL, Ayers RA, Bateman TA, Simske SJ. Bone development and age-related bone loss in male C57BL/6J mice. Bone 2003;33(3):387-398.

22. Almeida M, et al. Skeletal involution by age-associated oxidative stress and its acceleration by loss of sex steroids. $J$ Biol Chem 2007;282(37):27285-27297.

23. Ucer S, et al. The Effects of Aging and Sex Steroid Deficiency on the Murine Skeleton Are Independent and Mechanistically Distinct. J Bone Miner Res. 2017;32(3):560-574.

24. Nilsson ME, et al. Measurement of a Comprehensive Sex Steroid Profile in Rodent Serum by High-Sensitive Gas Chromatography-Tandem Mass Spectrometry. Endocrinology. 2015;156(7):2492-2502.

25. Jilka RL, O'Brien CA, Roberson PK, Bonewald LF, Weinstein RS, Manolagas SC. Dysapoptosis of osteoblasts and osteocytes increases cancellous bone formation but exaggerates cortical porosity with age. J Bone Miner Res. 2014;29(1):103-117.

26. Baron R, Tross R, Vignery A. Evidence of sequential remodeling in rat trabecular bone: morphology, dynamic histomorphometry, and changes during skeletal maturation. Anat Rec. 1984;208(1):137-145.

27. Willinghamm MD, Brodt MD, Lee KL, Stephens AL, Ye J, Silva MJ. Age-related changes in bone structure and strength in female and male BALB/c mice. Calcif Tissue Int. 2010;86(6):470-483.

28. Petkov PM, et al. An efficient SNP system for mouse genome scanning and elucidating strain relationships. Genome Res. 2004;14(9):1806-1811.

29. Bonewald LF. The amazing osteocyte. J Bone Miner Res. 2011;26(2):229-238

30. Jilka RL, O'Brien CA. The Role of Osteocytes in Age-Related Bone Loss. Curr Osteoporos Rep. 2016;14(1):16-25.

31. Miller RA, Buehner G, Chang Y, Harper JM, Sigler R, Smith-Wheelock M. Methionine-deficient diet extends mouse lifespan, slows immune and lens aging, alters glucose, T4, IGF-I and insulin levels, and increases hepatocyte MIF levels and stress resistance. Aging Cell. 2005;4(3):119-125.

32. Sun L, Sadighi Akha AA, Miller RA, Harper JM. Life-span extension in mice by preweaning food restriction and by methionine restriction in middle age. J Gerontol A Biol Sci Med Sci. 2009;64(7):711-722.

33. Yuan R, et al. Aging in inbred strains of mice: study design and interim report on median lifespans and circulating IGF1 levels. Aging Cell. 2009;8(3):277-287.

34. Glatt V, Canalis E, Stadmeyer L, Bouxsein ML. Age-related changes in trabecular architecture differ in female and male C57BL/6J mice. J Bone Miner Res. 2007;22(8):1197-1207.

35. Seeman E. Modeling and Remodeling. In: Bilezikian JP, Raisz LG, Martin TJ eds. Principles of Bone Biology. New York, NY: Academic Press; 2008:3-28.

36. Erben RG. Trabecular and endocortical bone surfaces in the rat: modeling or remodeling? Anat Rec. 1996;246(1):39-46.

37. Enlow DH. A study of the post-natal growth and remodeling of bone. Am J Anat. 1962;110:79-101.

38. Cadet ER, et al. Mechanisms responsible for longitudinal growth of the cortex: coalescence of trabecular bone into cortical bone. J Bone Joint Surg Am. 2003;85-A(9):1739-1748.

39. Price C, Herman BC, Lufkin T, Goldman HM, Jepsen KJ. Genetic variation in bone growth patterns defines adult mouse bone fragility. J Bone Miner Res. 2005;20(11):1983-1991.

40. Kavirayani AM, Sundberg JP, Foreman O. Primary neoplasms of bones in mice: retrospective study and review of literature. Vet Pathol. 2012;49(1):182-205.

41. Berndt A, et al. Genetic determinants of fibro-osseous lesions in aged inbred mice. Exp Mol Pathol. 2016;100(1):92-100.

42. Almeida M, O’Brien CA. Basic biology of skeletal aging: role of stress response pathways. J Gerontol A Biol Sci Med Sci. 2013;68(10):1197-1208.

43. Manolagas SC, Parfitt AM. What old means to bone. Trends Endocrinol Metab. 2010;21(6):369-374.

44. Jurk D, et al. Postmitotic neurons develop a p21-dependent senescence-like phenotype driven by a DNA damage response. Aging Cell. 2012;11(6):996-1004.

45. Kang C, et al. The DNA damage response induces inflammation and senescence by inhibiting autophagy of GATA4. Science. 2015;349(6255):aaa5612.

46. Neves J, Demaria M, Campisi J, Jasper H. Of flies, mice, and men: evolutionarily conserved tissue damage responses and aging Dev Cell. 2015;32(1):9-18.

47. Movérare-Skrtic S, et al. Osteoblast-derived WNT16 represses osteoclastogenesis and prevents cortical bone fragility fractures. Nat Med. 2014;20(11):1279-1288.

48. Maeda K, et al. Wnt5a-Ror2 signaling between osteoblast-lineage cells and osteoclast precursors enhances osteoclastogenesis. Nat Med. 2012;18(3):405-412. 
49. Okamoto M, et al. Noncanonical Wnt5a enhances Wnt/ $\beta$-catenin signaling during osteoblastogenesis. Sci Rep. $2014 ; 4: 4493$.

50. Li CY, Schaffler MB, Wolde-Semait HT, Hernandez CJ, Jepsen KJ. Genetic background influences cortical bone response to ovariectomy. J Bone Miner Res. 2005;20(12):2150-2158.

51. Baumann BD, Wronski TJ. Response of cortical bone to antiresorptive agents and parathyroid hormone in aged ovariectomized rats. Bone. 1995;16(2):247-253.

52. Li CJ, et al. MicroRNA-188 regulates age-related switch between osteoblast and adipocyte differentiation. J Clin Invest. 2015;125(4):1509-1522.

53. Schneider P, et al. Simultaneous 3D visualization and quantification of murine bone and bone vasculature using micro-computed tomography and vascular replica. Microsc Res Tech. 2009;72(9):690-701.

54. Pazzaglia UE, Sibilia V, Congiu T, Pagani F, Ravanelli M, Zarattini G. Setup of a bone aging experimental model in the rabbit comparing changes in cortical and trabecular bone: Morphological and morphometric study in the femur. J Morphol. 2015;276(7):733-747.

55. López-Otín C, Blasco MA, Partridge L, Serrano M, Kroemer G. The hallmarks of aging. Cell. 2013;153(6):1194-1217.

56. Hattner R, Frost HM. Mean skeletal age: its calculation, and theoretical effects on skeletal tracer physiology and on the physical characteristics of bone. Henry Ford Hosp Med Bull. 1963;11:201-216.

57. Farr JN, et al. Identification of Senescent Cells in the Bone Microenvironment. J Bone Miner Res. 2016;31(11):1920-1929.

58. van Deursen JM. The role of senescent cells in ageing. Nature. 2014;509(7501):439-446.

59. Todd H, et al. Wnt16 Is Associated with Age-Related Bone Loss and Estrogen Withdrawal in Murine Bone. PLoS ONE. 2015;10(10):e0140260.

60. Coppé JP, et al. Senescence-associated secretory phenotypes reveal cell-nonautonomous functions of oncogenic RAS and the p53 tumor suppressor. PLoS Biol. 2008;6(12):2853-2868.

61. Pivetta E, et al. MMP-13 stimulates osteoclast differentiation and activation in tumour breast bone metastases. Breast Cancer Res. 2011;13(5):R105.

62. Fujiwara Y, Piemontese M, Liu Y, Thostenson JD, Xiong J, O’Brien CA. RANKL (Receptor Activator of NFкB Ligand) Produced by Osteocytes Is Required for the Increase in B Cells and Bone Loss Caused by Estrogen Deficiency in Mice. J Biol Chem. 2016;291(48):24838-24850.

63. Piemontese M, Xiong J, Fujiwara Y, Thostenson JD, O'Brien CA. Cortical bone loss caused by glucocorticoid excess requires RANKL production by osteocytes and is associated with reduced OPG expression in mice. Am J Physiol Endocrinol Metab. 2016;311(3):E587-E593.

64. Thompson ML, Jimenez-Andrade JM, Mantyh PW. Sclerostin Immunoreactivity Increases in Cortical Bone Osteocytes and Decreases in Articular Cartilage Chondrocytes in Aging Mice. J Histochem Cytochem. 2016;64(3):179-189.

65. Baron R, Kneissel M. WNT signaling in bone homeostasis and disease: from human mutations to treatments. Nat Med. 2013;19(2):179-192.

66. Jilka RL, et al. Decreased oxidative stress and greater bone anabolism in the aged, when compared to the young, murine skeleton with parathyroid hormone administration. Aging Cell. 2010;9(5):851-867.

67. Almeida M, Ambrogini E, Han L, Manolagas SC, Jilka RL. Increased lipid oxidation causes oxidative stress, increased peroxisome proliferator-activated receptor-gamma expression, and diminished pro-osteogenic Wnt signaling in the skeleton. $J$ Biol Chem. 2009;284(40):27438-27448.

68. Iyer S, et al. FOXOs attenuate bone formation by suppressing Wnt signaling. J Clin Invest. 2013;123(8):3409-3419.

69. Kim HN, et al. DNA damage and senescence in osteoprogenitors expressing Osx1 may cause their decrease with age. Aging Cell. 2017;16(4):693-703.

70. Bentolila V, Boyce TM, Fyhrie DP, Drumb R, Skerry TM, Schaffler MB. Intracortical remodeling in adult rat long bones after fatigue loading. Bone. 1998;23(3):275-281.

71. Cardoso L, Herman BC, Verborgt O, Laudier D, Majeska RJ, Schaffler MB. Osteocyte apoptosis controls activation of intracortical resorption in response to bone fatigue. J Bone Miner Res. 2009;24(4):597-605.

72. Chesi A, et al. A Genomewide Association Study Identifies Two Sex-Specific Loci, at SPTB and IZUMO3, Influencing Pediatric Bone Mineral Density at Multiple Skeletal Sites. J Bone Miner Res. 2017;32(6):1274-1281.

73. Aged Rodent Colonies Handbook. National Institute on Aging Website. https://www.nia.nih.gov/research/dab/ aged-rodent-colonies-handbook/. Accessed August 8, 2017.

74. Jilka RL, O'Brien CA, Bartell SM, Weinstein RS, Manolagas SC. Continuous elevation of PTH increases the number of osteoblasts via both osteoclast-dependent and -independent mechanisms. J Bone Miner Res. 2010;25(11):2427-2437.

75. O'Brien CA, Jilka RL, Fu Q, Stewart S, Weinstein RS, Manolagas SC. IL-6 is not required for parathyroid hormone stimulation of RANKL expression, osteoclast formation, and bone loss in mice. Am J Physiol Endocrinol Metab. 2005;289(5):E784-E793.

76. Bouxsein ML, Boyd SK, Christiansen BA, Guldberg RE, Jepsen KJ, Müller R. Guidelines for assessment of bone microstructure in rodents using micro-computed tomography. J Bone Miner Res. 2010;25(7):1468-1486.

77. Baron R, Vignery A, Neff L, Silverglate A, Santa Maria A. Chapter 3. In: Recker RR, ed. Bone histomorphometry: techniques and interpretation. Boca Raton, FL: CRC Press, Inc.; 1983:13-35.

78. Dempster DW, et al. Standardized nomenclature, symbols, and units for bone histomorphometry: a 2012 update of the report of the ASBMR Histomorphometry Nomenclature Committee. J Bone Miner Res. 2013;28(1):2-17.

79. Sprent P, Smeeton MC. Applied Nonparametric Statistical Methods. Boca Raton, FL: Taylor \& Francis Group; 2007. 